\title{
HYDRODYNAMICAL MODELS OF TYPE II PLATEAU SUPERNOVAE
}

\author{
Melina C. Bersten ${ }^{1,2}$, Omar Benvenuto 3,4 , and Mario Hamuy ${ }^{1}$ \\ ${ }^{1}$ Departamento de Astronomía, Universidad de Chile, Casilla 36-D, Santiago, Chile; melina.bersten@ipmu.jp \\ 2 Institute for the Physics and Mathematics of the Universe, University of Tokyo, Kashiwanoha 5-1-5, Kashiwa, Chiba 277-8583, Japan \\ ${ }^{3}$ Facultad de Ciencias Astronómicas y Geofísicas, Universidad Nacional de La Plata, Paseo del Bosque s/n (B1900FWA) La Plata, Argentina \\ ${ }^{4}$ Instituto de Astrofísica de La Plata, IALP, CCT-CONICET-UNLP, Argentina \\ Received 2009 December 31; accepted 2010 December 26; published 2011 February 9
}

\begin{abstract}
We present bolometric light curves of Type II plateau supernovae obtained using a newly developed, one-dimensional Lagrangian hydrodynamic code with flux-limited radiation diffusion. Using our code we calculate the bolometric light curve and photospheric velocities of SN 1999em, obtaining a remarkably good agreement with observations despite the simplifications used in our calculation. The physical parameters used in our calculation are $E=1.25$ foe, $M=19 M_{\odot}, R=800 R_{\odot}$, and $M_{\mathrm{Ni}}=0.056 M_{\odot}$. We find that an extensive mixing of ${ }^{56} \mathrm{Ni}$ is needed in order to reproduce a plateau as flat as that shown by the observations. We also study the possibility to fit the observations with lower values of the initial mass consistently with upper limits that have been inferred from pre-supernova imaging of SN 1999em in connection with stellar evolution models. We cannot find a set of physical parameters that reproduce well the observations for models with pre-supernova mass of $\leqslant 12 M_{\odot}$, although models with $14 M_{\odot}$ cannot be fully discarded.
\end{abstract}

Key words: hydrodynamics - supernovae: general - supernovae: individual (SN 1999em)

Online-only material: color figures

\section{INTRODUCTION}

Type II plateau supernovae (SNe II-P) form a well-defined family characterized by a "plateau" in the optical light curve (LC; Barbon et al. 1979), where the luminosity remains nearly constant for a period of $\sim 100$ days, and the presence of prominent P-Cygni hydrogen lines in the spectrum. They constitute a subclass of the core-collapse $\mathrm{SNe}$ (CCSNe) - which includes Type Ib, Type Ic, and other subclasses of type II $\mathrm{SNe}$ - originated by the violent death of stars with initial masses greater than $8 M_{\odot}$ (Heger et al. 2003; Smartt et al. 2009) and sharing, in general terms, the same explosion mechanism. It has been shown that $\mathrm{SNe}$ II-P are the most common type of $\mathrm{SNe}$ in nature, amounting to $\sim 60 \%$ of all CCSNe. Apart from their astrophysical importance in connection with stellar evolution and the physics of the interstellar medium, additional interest on SNe II-P has recently arisen from the fact that they have been established as good distance indicators with potential application to cosmology, independent of Type Ia SNe (Hamuy \& Pinto 2002).

Massive stars may suffer considerable mass loss during early phases of their evolution, due to strong stellar winds or transfer to binary companions. Thus, they may lose part or all of their outermost envelope of unprocessed hydrogen and helium. Therefore, the vast diversity observed among CCSNe is related with the properties of the progenitor star. In the current picture, SNe II possibly have the least massive progenitors of all CCSNe subtypes that retain a significant fraction of their external hydrogen layers. This picture is combined with hydrodynamical models of SNe II-P, which show that a red supergiant progenitor with an extensive $\mathrm{H}$ envelope is necessary in order to reproduce the plateau-shaped LCs (Grassberg et al. 1971; Falk \& Arnett 1977; Chevalier 1976). Recent direct detection of the progenitors of several SNe II-P have confirmed this prediction (Van Dyk et al. 2003; Smartt et al. 2004). There is general agreement that the explosion of a massive star is originated by the collapse of its central parts into a neutron star or black hole when the iron core is formed at the end of the star's evolution, and further nuclear burning no longer provides thermal pressure to support the star. However, the mechanism of the energy deposition in the envelope still remains unknown in spite of the intensive theoretical modeling done in recent years (see Burrows et al. 2006; Janka et al. 2007, and references therein). The approach usually followed to model $\mathrm{SNe}$ is to decouple the explosion in two independent parts: (1) the core collapse and formation of the shock wave (SW) and (2) the ejection of the envelope. Based on the analysis of the propagation of the SW through the envelope, independently of how the shock is formed, it is possible to study the observational outcome of the explosion such as LCs and spectra. This approach has been extensively used (Falk \& Arnett 1977; Grassberg et al. 1971; Woosley 1988, among others) and it has led to the conclusion that the main factors influencing the outcome are the explosion energy and the progenitor structure.

Observationally, SNe II-P show a wide range of plateau luminosities $\left(L_{p}\right)$ and durations $\left(\Delta t_{p}\right)$, expansion velocities $\left(v_{\exp }\right)$, and nickel masses $\left(M_{\mathrm{Ni}}\right.$; Young \& Branch 1989; Hamuy 2001). The morphology of the LC of SNe II-P has been shown to be connected with physical properties of the progenitor object such as ejected mass $(M)$, explosion energy $(E)$, and pre-SN radius $(R)$. The relations between physical parameters and observables $\left(L_{p}, \Delta t_{p}, v_{\exp }\right)$ were first derived analytically by Arnett (1980) and then generalized by Popov (1993). Numerical calibrations of these relations were then given by Litvinova \& Nadezhin (1983, 1985, hereafter LN83 and LN85, respectively) based on a grid of hydrodynamical models for different values of $M, R$, and $E$. Hamuy (2003) and Nadyozhin (2003) applied such calibrations to a set of $\sim 20 \mathrm{SNe}$ II-P observations and thereby derived masses, radii, and explosion energies for their sample. However, these studies have not been fully satisfactory. As was noted in Hamuy (2001) and Bersten \& Hamuy (2009), some of the problems of inferring physical quantities from the relations of LN83 and LN85 are (1) the lack of good-quality data, (2) the use of simplified relations between ill-defined and hard-tomeasure photometric and spectroscopic parameters, and (3) the fact that some of the models are based on simplified physical 
assumptions. Some of the weaknesses of the LN83 and LN85 models are that they did not include the effect of nickel heating in their calculations, the use of old opacity tables, the neglect of any effect from line opacities, and the initial pre-SN models adopted. With the aim of addressing these problems and gaining a better knowledge of the pre-SN properties of SNe II-P, we have (1) enlarged the data set of spectra and LCs for 33 SNe II-P (M. Hamuy et al. 2011, in preparation) and (2) developed our own hydrodynamical model. This allows us to perform a comparison between models and data in a consistent way.

The development of our hydrodynamics code started in 2006 and is part of M.C.B.'s PhD thesis. In the interim since the beginning of the work there have been important advances in the field. For example, Utrobin (2007) performed a detailed analysis of SN 1999em and also studied how several physical parameters affect the LC. In that work, the author provided relations between physical and observed parameters that are valid only for SNe II-P with similar properties to those of SN 1999em. In addition, Kasen \& Woosley (2009) calculated a set of modeled LC and spectra of SNe II-P for different masses, metallicities, and explosion energies, using initial models obtained from stellar evolution calculations. They employed their models to describe the dependence of plateau luminosity and duration on explosion energy and progenitor mass. Nevertheless, the relations they found are simple and easy to apply only in the extreme case of no ${ }^{56} \mathrm{Ni}$ production. When ${ }^{56} \mathrm{Ni}$ is considered, the relations involve more parameters, which hinders their applicability to obtain physical parameters from observations. More recently, Dessart et al. (2010) studied the dependence of the properties of SN II-P ejecta on explosion energy and pre$\mathrm{SN}$ stellar evolution model. They found that the main-sequence mass of SNe II-P can be constrained using two measurements: (1) the photospheric velocity at 15 days after shock breakout, which is a good indicator of the explosion energy, and (2) the width of the nebular phase $\mathrm{O}_{\text {I }} \lambda \lambda 6303-6363$ line as an indicator of the helium-core mass and thereby of the mass of the star in the main sequence. Other works have also explored the effect of several physical parameters on the LC and other properties of SNe II-P (Chieffi et al. 2003; Young 2004), although without attempting to derive relations between physical and observed parameters. In recent years, there have been a number of works that analyzed the physical properties of individual SNe II-P based on hydrodynamical models (Baklanov et al. 2005; Utrobin \& Chugai 2009, among others).

In addition to the comparison with hydrodynamical models, there is an alternative way of deriving progenitor masses of $\mathrm{SNe}$ II-P. That is, using pre-SN images to search for the progenitor star, and measuring its brightness and color to derive a mass in connection with a stellar evolution model. Currently, there are three SNe II-P with a firm detection of the progenitor and $\sim 17$ without a positive detection (Smartt et al. 2009). The latter cases are still useful since they provide upper limits to the progenitor masses.

At this stage, there are three SNe II-P with hydrodynamical masses which have been studied in pre-SN imaging (namely, SN 1999em, SN 2004et, and SN 2005cs). As noted by Utrobin \& Chugai (2008) and Smartt (2009), in all cases the mass estimated by hydrodynamical models is higher than the estimate or upper limit given by pre-SN imaging. This discrepancy poses a very interesting and unsolved problem that is necessary to study.

In this work, we present our one-dimensional, flux-limited Lagrangian hydrodynamical code which is used for modeling bolometric LCs of SNe II-P. In general, the hydrodynamic mod- eling of SNe II-P is easier than the modeling of other CCSNe because the former explode in a very low density environment (Baron et al. 2000; Chevalier et al. 2006) and they possess extended, nearly spherically symmetric hydrogen envelopes, which smooth out possible inhomogeneities arising from differences in the explosion itself (Chevalier \& Soker 1989; Leonard \& Filippenko 2001). In addition, at least during the optically thick plateau phase, we expect a photosphere radiating as a "dilute" blackbody whose properties are mainly driven by the photospheric temperature (Eastman et al. 1996; Dessart \& Hillier 2005). We employ the usual hypothesis that the explosion can be decoupled into the collapse of the core and the ejection of the envelope. The energy which is transferred to the envelope (denoted by us as "explosion energy") plays the role of a coupling parameter between the internal and external problems. The processes that control the envelope ejection and the $\mathrm{SN}$ radiation do not depend on how the energy is transferred to the envelope as long as this process occurs during a short enough time and in a small enough region near the core. This means that for the purpose of modeling LCs it is possible to assume the core to be a point-like mass and energy source (Woosley \& Weaver 1994; Deputovich \& Nadyozhin 1999; Chieffi et al. 2003). Thus, our model is unable to describe details of the complex explosion mechanism.

The aim of developing our own code is to have available a simple, easy, and fast model to study, in a consistent way, the observed and physical parameters that determine the plateau phase for a sample of $33 \mathrm{SNe}$ II-P, which will be presented in a forthcoming paper. In this work, we present a detailed model for the prototypical SN II-P $1999 \mathrm{em}$, as a test case to show the consistency of the model. It will not be our goal in the future to craft specific models for each object, but to present a general study of the properties of the whole sample of SNe II-P.

A description of our numerical method, microphysics, and pre-SN models is given in Section 2.2. As a first step in our comparison with data, in Section 3 we analyze the case of the prototype SN 1999em, and we discuss how our model compares with previous hydrodynamical studies of this object. In Section 4, we test the feasibility of fitting the observations of SN 1999em with low-mass models, consistent with the upper limits obtained through pre-SNe imaging in connection with stellar evolution models. This analysis also shows the sensitivity of our bolometric LCs on the variation of physical parameters. Finally, in Section 5 we give our conclusions.

\section{CALCULATION METHOD}

Our SN models are computed by numerical integration of the hydrodynamical equations assuming spherical symmetry for a self-gravitating gas. The radiation transport is treated in the diffusion approximation with the flux-limited prescription of Levermore \& Pomraning (1981). The explosion is simulated by injecting a certain amount of energy during a very short time as compared with the hydrodynamic timescale near the center of the progenitor object. This energy induces the formation of a powerful SW that propagates through the progenitor transforming thermal and kinetic energy of the matter into energy that can be radiated from the stellar surface. To calculate SWs, we include, as usual, an artificial-viscosity term in the equations of moment and energy.

The equations and numerical method used are discussed in Section 2.1. In Section 2.2, we give a brief description of the constitutive relations used in our code. The energy deposited by radioactive decay is discussed in Section 2.3. The initial models 
are described in Section 2.4. A discussion of the approximations used in our calculations is given in Section 2.5. Finally, in Section 2.6 we describe which parameters of the model are suitable to compare with observations.

\subsection{Equations for Radiation Transport and Hydrodynamics}

Our code follows a one-dimensional, Lagrangian prescription that solves for the variables: radius $(r)$, velocity $(u)$, density $(\rho)$, and temperature $(T)$ as a function of the Lagrangian mass coordinate $m$, using the following equations which simply express conservation laws:

$$
\begin{gathered}
\frac{\partial r}{\partial t}=u, \\
V=\frac{1}{\rho}=\frac{4 \pi}{3} \frac{\partial r^{3}}{\partial m}, \\
\frac{\partial u}{\partial t}=-4 \pi r^{2} \frac{\partial}{\partial m}(P+q)-\frac{G m}{r^{2}}, \\
\frac{\partial E}{\partial t}=\epsilon_{\mathrm{Ni}}-\frac{\partial L}{\partial m}-(P+q) \frac{\partial V}{\partial t}, \\
L=-\left(4 \pi r^{2}\right)^{2} \frac{\lambda a c}{3 \kappa} \frac{\partial T^{4}}{\partial m} .
\end{gathered}
$$

Here, $V$ is the specific volume, $P$ is the total pressure (of gas and radiation), and $q$ is the artificial viscosity, which is included in the equations to spread the pressure and energy over several mass zones at the shock front. There are many expressions for the artificial viscosity, all dependent on the velocity gradient, which aim at providing a convenient interpolation scheme between unshocked and shocked fluid. We adopt the expression given by Von Neumann \& Richtmyer (1950). $E$ is the internal energy per unit of mass (of gas and radiation) and $\epsilon_{\mathrm{Ni}}$ is the energy deposited by the radioactive decay of nickel as we describe in Section 2.3. We do not consider other sources of cooling or heating in Equation (1d), such as losses due to neutrino processes or energy released by thermonuclear reactions. Even if neutrinos are very important in the formation of the SW, as the explosion depends noticeably on the efficiency of their energy transfer, most of them are emitted before the SW reaches the stellar photosphere, so they have no effect on later epochs of the SN evolution (see Hillebrandt 1994; Burrows 1991; Janka et al. 2007). The energy released by explosive nucleosynthesis is much less than the energy of the SW, as has been previously established (see Imshenik \& Nadezhin 1965; Woosley \& Weaver 1994; Arnett 1996). $T$ is the temperature of both matter and radiation, $\kappa$ is the Rosseland mean opacity, and $L$ is the luminosity. Finally, $\lambda$ is the so-called flux-limiter, included in the equation of radiative transfer in the diffusion approximation to ensure a smooth transition between diffusion and free-streaming regimes to assure causality. The expression adopted for $\lambda$ is

$$
\lambda=\frac{6+3 R}{6+3 R+R^{2}},
$$

where

$$
R=\frac{\left|\nabla T^{4}\right|}{\kappa \rho T^{4}}=\frac{4 \pi r^{2}}{\kappa T^{4}}\left|\frac{\partial T^{4}}{\partial m}\right| .
$$

The quantities $E, P, q$, and $\kappa$ are functions of $\rho, T$, and chemical composition. Further details on these relations are given in Section 2.2.
As boundary conditions we use $u=0$ near the center (at $m=M_{\text {core }}$, where typically we adopt $\left.M_{\text {core }}=1.4 M_{\odot}\right)$, and $P_{\text {gas }}=\rho=0$ at the surface $(m=M)$.

We discretize the previous equations using a space-centering discretization with the extensive quantities evaluated at the interfaces and the intensive quantities in the midpoints of the grid zones. Two time steps are adopted in each cycle: one to advance the velocity, and the other to advance the material state variables. The time step is chosen depending on limitations of stability and accuracy (see the discussion below). Special care is taken in the centering of the opacity used in the discretization of Equation (1e) in order to prevent numerical noise from appearing due to the propagation of the radiation flow at the steep front where the opacity changes significantly (Christy 1967).

We use an explicit scheme for the integration of the hydrodynamic equations, but a semi-implicit scheme for the temperature, similar to the one used by Falk \& Arnett (1977). The equations are linearized in $\delta T$ and solved iteratively for each time step using the tridiagonal method. The discretization typically uses 300 mesh points, with a finer sampling for the outer layers typically smaller than $10^{-6} M_{\odot}$. This value was chosen based on tests performed with our model and following previous works (Woosley 1988; Ensman \& Burrows 1992) that show that the early LC is sensitive to the mass zoning in the outer layers when a coarse grid is used.

Given that we are using an explicit hydrodynamic scheme, the time step should be chosen as a fraction of the minimum of the Courant condition for all zones in order to achieve stability. We note that we have also imposed additional conditions on the time step: we have required that changes in temperature, density, and flux over one time step be less than $5 \%$.

The formation of the SW following core collapse is simulated by artificially adding internal energy ("thermal bomb") almost instantaneously in the central region of the core. We usually set a mass cut of $1.4 M_{\odot}$ which is the material assumed to collapse and form a neutron star or black hole. Specifically, we employ an exponential function both in mass and in time to distribute the injected energy across several layers and some time steps, which helps to improve the numerical treatment. With the scale factors used in this work for the exponential functions almost all of the explosion energy was completely injected in a shell of $0.1 M_{\odot}$ and within less than 1 minute, i.e., a short time compared with the hydrodynamical timescale. Although there may be some differences in the velocity and temperature profiles during the shock propagation on the scale factors used, our values were chosen such that the differences in the velocity profiles using this value or lower values were not significant. We have also tested explosions generated by injecting kinetic energy and we have obtained similar results. The latter method, however, leads to very short numerical time steps, and therefore to slower calculations. We have checked the accuracy of our calculation method by testing the conservation of energy. The total amount of energy is conserved within $0.6 \%$ but if we consider the conservation of energy between two consecutive time steps, this is even better, within $4 \times 10^{-6}$. We consider it very acceptable for our purposes.

\subsection{Input Physics}

The equation of state (EOS) is calculated using simple expressions for $T, \rho$, composition, and for the ionization degrees of hydrogen and helium corresponding to local thermodynamical equilibrium (LTE). Therefore, the degree of ionization is determined by solving the corresponding set of Saha equations 
for ionization of hydrogen and the first and second ionization of helium. Ionization of heavy elements is neglected in our EOS (but, of course, it is taken into account in the calculation of the opacity). The degeneracy pressure is also included in the EOS. We tested our results using a more sophisticated EOS, such as the one available from the Los Alamos Tables (Rogers et al. 1996), and we did not find any significant differences with respect to the results obtained using our simple EOS. This is expected because of the low densities attained in most of the layers of the models.

The Rosseland mean opacities, $\kappa$, used in our calculations are derived using the OPAL opacity tables (Iglesias \& Rogers 1996, and references therein). As these tables are given for $T>6 \times$ $10^{3} \mathrm{~K}$, we complement them with the opacity table provided by Alexander \& Ferguson (1994) for lower temperatures, which includes molecular opacities. The tables are interpolated to each other in order to guarantee a smooth transition at $T=10^{4} \mathrm{~K}$.

These tables allow us to calculate opacities for several metallicities. Also, for a fixed metallicity, different mixtures of $\mathrm{H}, \mathrm{He}, \mathrm{C}$, and $\mathrm{O}$ can be used. Although in this work we adopt a fixed value of $Z=0.02$, departures from this value (as expected in the inner regions of the object) are taken into account as excesses of $\mathrm{C}$ and $\mathrm{O}$ with respect to the values of the adopted metallicity, at the expense of He.

Figure 1 shows the opacity given by the tables as a function of temperature for $Z=0.02$ and different densities. The range of temperature shown corresponds to the values reached during the evolution of an SN II-P while the values of density are restricted to those achieved during the plateau phase. Also shown in the plot is the contribution to the opacity from electron scattering. Note that electron scattering is the dominant source of opacity for $T>10^{4} \mathrm{~K}$ and $\rho<10^{-10} \mathrm{~g} \mathrm{~cm}^{-3}$.

The Rosseland mean opacity includes scattering and absorption processes. Scattering is the dominant process in the SN ejecta during the plateau phase when the densities are $\rho<10^{-10}$ (see Figure 1), and this will be so until most of the electrons recombine with ions and absorption processes become an important source of opacity. On the other hand, in rapidly expanding envelopes where large velocity gradients are present, the Rosseland mean opacity underestimates the true line opacity (Karp et al. 1977), which hinders the estimation of the actual opacity in the outermost (recombined) layers. Another effect that is not included in the calculation of $\kappa$ is the non-thermal excitation or ionization of electrons that are created by Compton scattering of $\gamma$-rays emitted by radioactive decay of ${ }^{56} \mathrm{Ni}$ and ${ }^{56} \mathrm{Co}$. The LTE ionization used in the calculation of $\kappa$ considerably underestimates the true ionization. The correct way to treat these effects is to calculate the actual contribution of nonthermal ionization to the opacity and to include the expansion opacity of lines. However, such treatment is beyond the scope of this paper. We adopt an alternative approach to the problem that has been extensively used in the literature which consists in using a minimum value of the opacity (or "opacity floor") to partially solve the shortcoming in the Rosseland mean opacity. Given the dependence of the opacity on composition, usually two values of the opacity floor are used: one for the H-rich envelope material and another for the metal-rich core. The opacity at each time and mesh point is chosen as the maximum value between the tabulated Rosseland mean opacity and the opacity floor for the corresponding composition.

Values of the opacity floor should be based on contributions to the opacity that are not included in the Rosseland mean opacity tables. There are differences in the values adopted

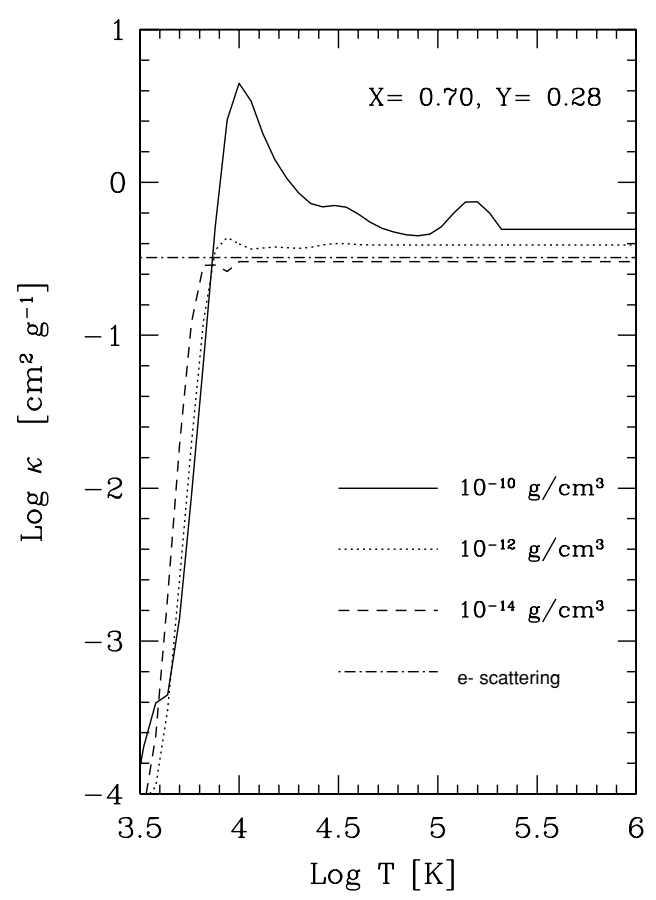

Figure 1. Run of the Rosseland opacity on temperature $(T)$ and density $(\rho)$ for solar metallicity $(Z=0.02)$ as used in our calculation without including any "opacity floor" (see discussion in Section 2.2). The ranges of $T$ and $\rho$ shown are typical for SNe II-P. The hydrogen $(X)$ and helium $(Y)$ mass fractions used are indicated. We also include the electron scattering opacity (considering full ionization, which is certainly unrealistic for the low-temperature sector of this plot) in order to show the dominance of this source for $T>10^{4} \mathrm{~K}$ and $\rho<10^{-10} \mathrm{~g} \mathrm{~cm}^{-3}$. Note that this value of the density is reached early on in the $\mathrm{SNe}$ evolution. For lower temperatures the absorption processes become an important source of opacity.

in the literature. For example, Herzig et al. (1990) adopted a value of $0.01 \mathrm{~cm}^{2} \mathrm{~g}^{-1}$ for the whole structure. Shigeyama $\&$ Nomoto (1990) used minimum values for the bound-free and bound-bound opacities of $k_{\mathrm{bf}}=9 \times 10^{-3} Y \mathrm{~cm}^{2} \mathrm{~g}^{-1}$ and $k_{\mathrm{bf}}=1 \times 10^{-2} Z \mathrm{~cm}^{2} \mathrm{~g}^{-1}$, where $Y$ and $Z$ denote the mass concentration of helium and heavy elements. Note that it is needed to add scattering and free-free absorption effects to the latter in order to obtain the Rosseland mean opacity. Swartz et al. (1991) employed $0.05 \mathrm{~cm}^{2} \mathrm{~g}^{-1}$ for the envelope material, and $0.1 \mathrm{~cm}^{2} \mathrm{~g}^{-1}$ for the metal-rich core material. And Young (2004) used a value of $0.25 \mathrm{~cm}^{2} \mathrm{~g}^{-1}$ for the helium-rich core, and $0.01 \mathrm{~cm}^{2} \mathrm{~g}^{-1}$ for the hydrogen-rich envelope. We do not intend to be exhaustive but the examples above provide a summary of the values adopted in the field of SNe II.

In this work, the minimum opacity values adopted are $0.01 \mathrm{~cm}^{2} \mathrm{~g}^{-1}$ for the envelope material, and $0.24 \mathrm{~cm}^{2} \mathrm{~g}^{-1}$ for the metal-rich core material. These values were chosen based on a comparison performed between our code and the STELLA code (Blinnikov \& Bartunov 1993; Blinnikov et al. 1998) using the same initial model provided by Umeda \& Nomoto (2005). Note that STELLA is an implicit hydrodynamic code that incorporates multi-group radiative transfer, and which additionally uses different opacity tables and includes the effect of line opacities (Sorokina \& Blinnikov 2002). In spite of our approximations, it is important to remark that we obtain an excellent overall agreement of the LC given by both codes (both in terms of the duration of the plateau and of the morphology of the bolometric LC) when the opacity floor is set the values given above. 


\subsection{Gamma-ray Deposition}

The radioactive decay of ${ }^{56} \mathrm{Ni} \rightarrow{ }^{56} \mathrm{Co} \rightarrow{ }^{56} \mathrm{Fe}$ constitutes a relevant source of energy that heats the envelope of the SN. Several studies of SNe II-P have usually ignored the contribution of such a component during the early $\mathrm{SN}$ evolution and have only considered it beyond the end of the plateau phase. Such an approach would be correct only if ${ }^{56} \mathrm{Ni}$ were very deeply concentrated in the ejecta, in which case the local deposition of gamma rays is a good approximation because gamma photons can hardly diffuse out from the regions where they are formed. Nevertheless, as was shown in studies of SN 1987A, there is no reason to assume this type of ${ }^{56} \mathrm{Ni}$ distribution (Shigeyama et al. 1988; Woosley et al. 1988; Arnett 1988; Blinnikov et al. 2000, among others) and therefore we need to calculate the diffusion of gamma rays from the location where they are emitted to the outer regions. To calculate this, we solve the gamma-ray transfer in the gray approximation for any distribution of ${ }^{56} \mathrm{Ni}$, assuming that gamma rays interact with matter only through absorption. It has been shown by comparison with Monte Carlo simulations of Type Ia $\mathrm{SNe}$ that the complex scattering process between gamma rays and electrons can be satisfactorily approximated as an absorptive process (Sutherland \& Wheeler 1984; Swartz et al. 1995). The value for the gamma-ray opacity that we adopt here is $\kappa_{\gamma}=0.06 y_{e} \mathrm{~cm}^{2} \mathrm{~g}^{-1}$, where $y_{e}$ is the number of electrons per baryon.

The rate of energy per gram released by the $\mathrm{Ni}-\mathrm{Co}-\mathrm{Fe}$ decay is

$$
\begin{aligned}
\epsilon_{\mathrm{rad}}= & 3.9 \times 10^{10} \exp \left(-t / \tau_{\mathrm{Ni}}\right)+6.78 \times 10^{9}\left[\exp \left(-t / \tau_{\mathrm{Co}}\right)\right. \\
& \left.-\exp \left(-t / \tau_{\mathrm{Ni}}\right)\right] \mathrm{erg} \mathrm{g}^{-1} \mathrm{~s}^{-1}
\end{aligned}
$$

where $\tau_{\mathrm{Ni}}=8.8$ days and $\tau_{\mathrm{Co}}=113.6$ days are the mean lifetimes of the radioactive isotopes. The amount of energy deposited at each point is given by the solution of the gamma-ray transfer multiplied by the previous expression. In Figure 2, we show the gamma-ray deposition ${ }^{5}$ for the case of a polytrope with index $n=3$, initial mass of $10 M_{\odot}$, and different initial radii, assuming a constant distribution of ${ }^{56} \mathrm{Ni}$ up to $3 M_{\odot}$. Note that the diffusion of gamma rays from the region where they form becomes more noticeable as the object becomes more extended and diluted.

The possibility to use an arbitrary distribution of ${ }^{56} \mathrm{Ni}$ allows us to study different types of mixing and their effect on the LC. We have found, as shown in Section 3.1.4, that radioactivity becomes an important source of energy even during the plateau phase if we allow extensive ${ }^{56} \mathrm{Ni}$ mixing.

\subsection{Initial Models}

There are two different types of initial (or pre-SN) models: those coming from stellar evolution calculations (or "evolutionary" models), and those from non-evolutionary calculations where the initial density and chemical composition are parameterized in a convenient way. In this work, we use double polytropic models in hydrostatic equilibrium as non-evolutionary pre-SN models. Although a single polytrope may represent very well the envelope of the pre-SN object, the inner, dense part that is expected for this type of object is not well reproduced. One way to improve this situation is to consider two polytropes:

\footnotetext{
5 The deposition of gamma rays is defined by the energy deposited in each point normalized by the value corresponding to complete thermalization at the same location where the gamma rays are emitted.
}

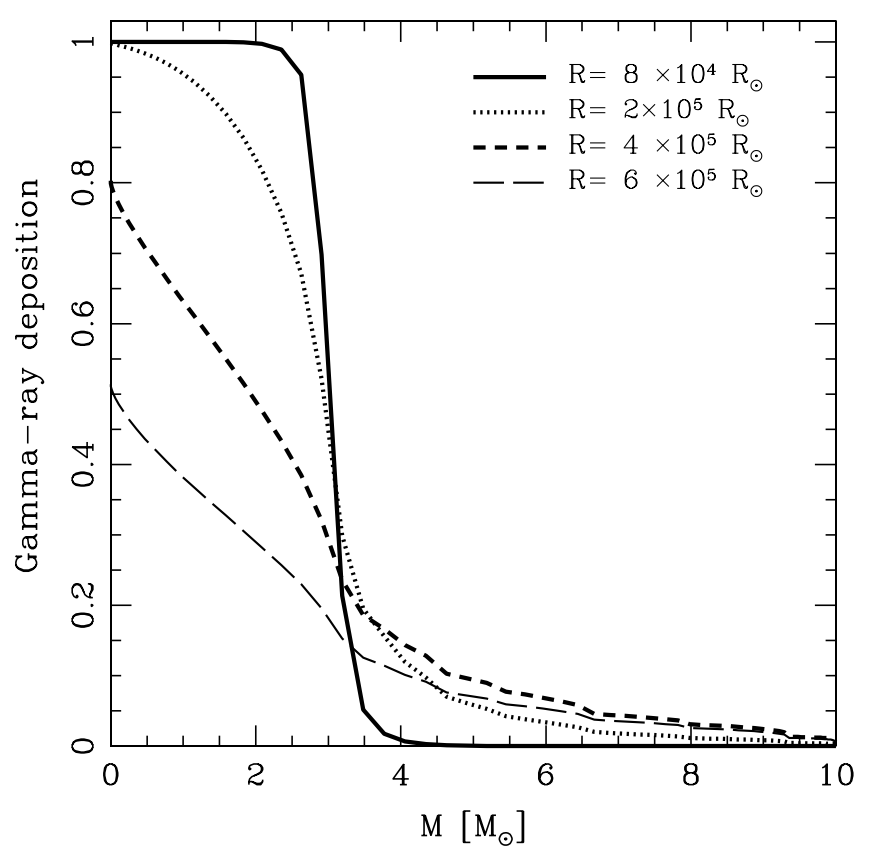

Figure 2. Gamma-ray deposition as a function of mass for a polytrope with index $n=3$, initial mass of $10 M_{\odot}$, and different initial radii for a constant distribution of ${ }^{56} \mathrm{Ni}$ up to $3 M_{\odot}$. The diffusion of the gamma rays out of the region where they form becomes more noticeable as the object gets more diluted.

one representing the inner dense core, and the other accounting for the outer extended envelope. As initial composition we use parametric chemical profiles with mixing between layers of different chemical composition (see Figure 4 in Section 3.1 for SN 1999em). Mixing is expected to occur during the explosion due to hydrodynamical instabilities which can carry the hydrogen very deep into the core and the ${ }^{56} \mathrm{Ni}$ out into the hydrogen envelope as previous studies of SN 1987A have shown (Dotani et al. 1987; Shigeyama et al. 1988; Woosley et al. 1988; Arnett 1988; Haas et al. 1990; Blinnikov et al. 2000, among others). Unfortunately, we cannot properly take into account this effect in our one-dimensional prescription. To ameliorate this we implicitly include this effect by imposing mixing in our initial chemical profile. It is important to note that even if mixing is expected only after the shock propagation, the effect on the dynamics of imposing it at the initial time is not noticeable until the shock breakout, when the recombination front recedes into the ejecta.

In order to calculate numerically a double polytropic model, it is necessary to solve the equations of hydrostatic equilibrium and mass conservation assuming a polytropic approximation where the pressure has a dependence on density of the form: $P=K \rho^{\gamma}=K \rho^{n /(n+1)}$, where $K$ is a constant and $n$ is the polytropic index. Two different polytropic relations with different indices $\left(n_{i}\right.$ and $\left.n_{o}\right)$ and constants $\left(K_{i}\right.$ and $\left.K_{o}\right)$ are adopted to mimic the characteristic structure of a red supergiant. To calculate the composite polytrope, the previous equations are numerically integrated outward from the inner border (stellar core) to a pre-selected point (fitting point) using the internal polytropic relation. A second inward integration is done from the outer border (stellar surface) to the fitting point using the external polytropic relation. The problem is equivalent to a twopoint boundary condition for the case where there are unknown free parameters at both ends of the domain. In our case, the free parameters are $n_{i}, K_{i}$, and $K_{o}$, while the external index is fixed to $n_{o}=3$. Starting from initial guesses, the values of the 
free parameters are iteratively sought so that the solution joins smoothly at the fitting point. This process is performed using a shooting method. Once the values of $n_{i}, K_{i}$, and $K_{o}$ are found, the density (or pressure) distribution and mass distribution can be calculated. The initial temperature profile is calculated in an iterative fashion using our EOS to ensure hydrostatic equilibrium. This prevents the formation of a spurious SW.

Using this method, a variety of initial models in hydrodynamical equilibrium can be calculated by changing the fitting point and the boundary conditions for a given mass, radius, and central density, which allows us to study how the internal structure of the initial model affects the LC.

Alternatively to the parametric initial model, we have tested our code using initial models from different stellar evolution calculations. We found several features in the resulting LC that are not present in the observations, as was previously noted in the literature (see Utrobin \& Chugai 2008). Specifically, several bumps and wiggles appear during the plateau phase. Regardless of the differences among evolutionary models, all of them have the common characteristic of showing a sharp boundary between layers with different chemical composition and a steeper jump in density between the helium core and the hydrogen envelope than in our double polytropic models. These two characteristics are the ones responsible for the unobserved bumps in the LC. We note that a possible reason for the relatively poor results we obtained from evolutionary initial models may be the use of one-dimensional calculations, which cannot take into account effects that produce mixing, such as Rayleigh-Taylor (R-T) instabilities. This point deserves further scrutiny, although it is beyond the scope of this paper.

In summary, we decided to use double polytropic initial models based on the following facts. (1) We have generally found a better agreement with observations using our parameterized profiles, in accordance to previous studies (Utrobin 1993; Baklanov et al. 2005; Utrobin 2007, among others). (2) There is a limited set of pre-SN models from stellar evolutionary calculations available to us. (3) Parameterized initial models allow us to easily vary physical properties and study their effect on the LC, which is critical for our goal of studying a large sample of SNe. However, we emphasize that the choice of polytropic initial models makes the connection with the structure of a progenitor star difficult to assess.

\subsection{Approximations}

Several approximations are made in the equations of radiation hydrodynamics. We assume that the fluid motion can be described by one-dimensional, radially symmetric flow. The explosion mechanism of CCSNe is not well known but it may be a very asymmetric process. However, for this particular subtype of SNe with very extended hydrogen envelopes, the asymmetries expected from the explosion mechanism itself appear to be smoothed. This is supported by recent spectropolarimetric studies (Leonard \& Filippenko 2005).

We use the equilibrium diffusion approximation to describe the radiative transfer. This approximation assumes that radiation and matter are strongly coupled with a single characteristic temperature and a spectral energy distribution described by a blackbody function (BB hereafter). The approximation breaks down at shock breakout and at late phases when the ejecta is completely recombined and the object becomes transparent. Fortunately, during the plateau phase-which is our main interest-this approximation is adequate. Also note that nonLTE calculations of SN II spectra show that deviations from
LTE have significant effects on the lines but not on the overall continuum (Baron et al. 1996; Dessart \& Hillier 2008). At late phases, for $\mathrm{SNe}$ that experience little interaction with the interstellar medium, as is the case of SNe II-P, the bolometric luminosity can be simply approximated as the luminosity deposited by the radioactive decay of ${ }^{56} \mathrm{Co}$, at least during the early part of the radioactive tail. This is supported by the fact that the luminosity declines obeying an exponential law with a very similar rate to that of the decay of ${ }^{56} \mathrm{Co}$. There was no attempt to apply our model beyond $\sim 180$ days.

During the transition between plateau and radioactive tail, the envelope is fully recombined and the notion of a photosphere loses meaning. Thus, this transition regime is poorly described with our radioactive transfer prescription and therefore a detailed description of this phase cannot be assessed here. As stated above, we have performed a comparison of our calculations with those of the STELLA code, obtaining an excellent agreement between the bolometric luminosities derived by both methods. This is very satisfactory considering that the STELLA code involves a more sophisticated treatment of the radiative transfer by solving these equations using a multi-group prescription and including the effect of line opacities (Blinnikov \& Bartunov 1993; Sorokina \& Blinnikov 2002).

\subsection{Comparison with Observables}

One of the main motivations for this work is the unprecedented database of $B V I$ LCs of $~ 30$ SNe which offers the opportunity to significantly improve our understanding of SNe II-P and their progenitors. Given that our code produces bolometric LCs, we need to compute bolometric luminosities for our data set. In Bersten \& Hamuy (2009), we found a tight correlation between bolometric correction and colors, which allowed us to calculate bolometric luminosities from $B V I$ colors with an uncertainty of only $\sim 0.05$ dex. ${ }^{6}$

Another critical parameter to compare with observations is the photospheric velocity yielded by our models. Expansion velocity estimates from the minimum of several spectral lines $\left(\mathrm{H}_{\alpha}, \mathrm{Fe}\right.$ II $\lambda 5169, \mathrm{H}_{\beta}$, and $\left.\mathrm{H}_{\gamma}\right)$ of our data set were given by Jones et al. (2009). Since each line forms at a different shell, it is not straightforward to compare the observed velocities with photospheric velocities. Jones et al. (2009) got around this problem and derived congruent calibrations between the velocity derived from the absorption minimum of such lines and the photospheric velocity using two independent atmosphere models (Eastman et al. 1996 and Dessart \& Hillier 2005; E96 and D05, hereafter, respectively). As noted by Jones et al. (2009), $\mathrm{H}_{\beta}$ provides a very good proxy to the photosphere velocity as it is not highly saturated as $\mathrm{H}_{\alpha}$, and is present over most of the $\mathrm{SN}$ evolution. Therefore, in the comparisons with our models, we chose to use the calibration given by Jones et al. (2009) for this particular line. However, in spite of the satisfactory behavior of this calibration in the high-velocity regime $\left(v \gtrsim 5000 \mathrm{~km} \mathrm{~s}^{-1}\right)$, for lower velocities the atmosphere models fail to reproduce the behavior shown by the data (see Figure 9 of Jones et al. 2009). Thus, we decided to include also in our comparison the velocities estimated from the Fe II $\lambda 5169$ line, which is present

\footnotetext{
$6 \quad$ An alternative would be to compute $B V I$ LCs from our models but the only available atmosphere model that we have at this point is the blackbody spectral distribution. However, as several studies have shown and as can be seen in Figure 3 of Bersten \& Hamuy (2009), the luminosity of SNe II-P depart considerably from a BB behavior very early in the SN evolution, which hampers the comparison.
} 
at later phases of the $\mathrm{SN}$ evolution when the velocities are below the limit imposed by the calibration of hydrogen lines.

For consistency with the atmosphere models, we define the photospheric position as the layer where the total continuum optical depth is $\tau=2 / 3$. Note that to calculate $\tau$ we do not include the opacity floor because such a minimum is only included to take into account line effects, and therefore is a bound-bound opacity that does not contribute to form the continuum. With this definition, the photosphere follows the recombination wave (RW) as we show in Section 3.1.3. Note that this behavior of the photosphere is due to the fact that electron scattering is the dominant source of opacity (see Figure 1). Had we included the opacity floor in the definition of $\tau$, the $\tau=2 / 3$ surface would be pushed well above the recombination front.

We remark that with this definition, the photosphere is essentially the surface of last scattering. However, the surface where the continuum is actually formed is located in a deeper layer called the "thermalization depth" and this is due to the dominance of the electron scattering over the absorption processes (Sobolev 1980; Hoflich 1991; Montes \& Wagoner 1995). With our simple prescription of radiative transfer we cannot accurately determine the location of the thermalization depth because there radiation decouples from matter. Note that the color temperature, determined by a blackbody fit to the broadband photometry, is nearly coincident with the temperature of the thermalization depth but is greater than the effective temperature defined by $T_{\mathrm{eff}}=L / 4 \pi \sigma R_{\mathrm{ph}}^{2}$, where $R_{\mathrm{ph}}$ is the photospheric radius. In Bersten \& Hamuy (2009), we derived a calibration between $T_{\text {eff }}$ and color based on the E96 and D05 models. Using this calibration we study the evolution of $T_{\text {eff }}$ for our sample of SNe II-P and we compare it with $T_{\text {eff }}$ derived from our code.

\section{APPLICATION TO SUPERNOVA $1999 \mathrm{em}$}

In preparation for our comparison with the sample of SNe II-P in a forthcoming paper, we analyze here the case of the prototype SN 1999em to show the details of how our code works. The choice of this object was motivated by the fact that this is one of the best observed SNe of its type both in terms of wavelength coverage and temporal sampling. In addition, SN 1999em was previously modeled with two different hydrodynamical codes (Baklanov et al. 2005; Utrobin 2007), which provided consistent physical parameters of the pre-SN model. Here, we start our study using our code and initial parameters consistent with such studies (Section 3.1). In Section 3.2, we discuss how our results compare with previous studies of SN 1999em.

It is interesting to note that currently there is an unsolved discrepancy between the mass of the progenitor object derived from hydrodynamical models, and estimates from pre-SN images in connection with stellar evolution models (Utrobin \& Chugai 2008; Smartt et al. 2009; Smartt 2009). For example, for SN $1999 \mathrm{em}$ the hydrodynamical mass is close to $19 M_{\odot}$ (this corresponds to the mass of the progenitor at the time of explosion, so the zero-age main-sequence star would be even greater) while from pre-SN images an upper limit of $15 M_{\odot}$ was derived for the progenitor star at the ZAMS. In order to address this problem, in Section 4 we study the possibility of fitting the LC and velocity evolution of SN 1999em with lower values of the envelope mass.

SN 1999em was discovered shortly after the explosion on 1999 October 29 UT by the LOSS program (Li 1999). Based on the Expanding Photosphere Method (EPM) study by Jones et al. (2009), we assume SN 1999em exploded 3 days before discovery. This is consistent with the constraint imposed by a negative detection (limiting magnitude 19) on an image obtained on 1999 October 20 UT. In order to compare with our model, we corrected the times elapsed since explosion by time dilation based on the redshift of the host galaxy. We adopted the Cepheid distance of 11.7 Mpc given by Leonard et al. (2003) to compute bolometric luminosities as explained in Bersten \& Hamuy (2009) using photometric data obtained at Cerro Tololo InterAmerican Observatory (CTIO), Las Campanas Observatory (LCO), and the European Southern Observatory (ESO) at La Silla (Hamuy 2001).

\subsection{High-mass SN $1999 \mathrm{em}$}

In this section, we use a pre-SN model with initial parameters consistent with the optimal hydrodynamical model of Utrobin (2007). Specifically, we adopt an initial mass of $19 M_{\odot}$, radius of $800 R_{\odot}$, and explosion energy of $E=1.25$ foe ( 1 foe $=1 \times$ $\left.10^{51} \mathrm{erg}\right)$. This energy was released as thermal energy near the core of the object in a very short timescale as compared with the hydrodynamic timescale of our model. We also assume a nickel mass of $0.056 M_{\odot}$ which was determined by the luminosity of the radioactive tail. This parameter is quite different from the nickel mass of $0.036 M_{\odot}$ used by Utrobin (2007), which was determined using the quasi-bolometric luminosity given by Elmhamdi et al. (2003). Their luminosity was based on the integration of only $U B V R I$ photometry with a constant value of 0.19 dex added to take into account the infrared luminosity, while our bolometric luminosity is based on a quantitative study of the bolometric correction for SNe II-P (see Section 2.5). Note also that our value for the nickel mass is closer to the estimate of $0.06 M_{\odot}$ given by Baklanov et al. (2005). In the following analysis, we denote this model as M19R8E1.25NI56.

In our calculations, we remove the central $1.4 M_{\odot}$ which is assumed to form a neutron star. The initial density profile as a function of mass and radius for model $\mathrm{M} 19 \mathrm{R} 8 \mathrm{E} 1.25 \mathrm{NI} 56$ is shown in Figure 3. Note that the initial structure is composed of a dense core and an extended envelope characteristic of a red supergiant. The chemical composition profiles are shown in Figure 4 . The outer parts of the envelope, $M>9 M_{\odot}$, have a homogeneous composition with mass fractions of $X=0.735$, $Y=0.251$, and $Z=0.02$. From there inward, hydrogen and helium are mixed in order to prevent a sharp boundary between the H-rich and the He-rich layers. Such sharp boundaries are characteristic of stellar evolution models but fail to reproduce the observations. Note that we allow $\mathrm{H}$ to mix very deep inside the core and ${ }^{56} \mathrm{Ni}$ is mixed out in the envelope until $\sim 15 M_{\odot}$. This type of mixing is presumably due to R-T instabilities that occur behind the shock front, as is obtained in multi-dimensional hydrodynamic calculations (Mueller et al. 1991; Kane et al. 2000) and supported by studies of SN 1987A (Shigeyama et al. 1988; Woosley et al. 1988; Arnett 1988; Blinnikov et al. 2000, among others). The presence of $\mathrm{H}$ in the core leads to a smooth transition between the plateau and the radioactive tail. The distribution of ${ }^{56} \mathrm{Ni}$ to external layers helps to reproduce a plateau as flat as that observed in SN 1999em (see Section 3.1.3).

Figure 5 shows a comparison between the bolometric LC (solid line) obtained with our code for model M19R8E1.25NI56, and the observations of SN 1999em (dots). The luminosity due to ${ }^{56} \mathrm{Ni} \rightarrow{ }^{56} \mathrm{Co} \rightarrow{ }^{56} \mathrm{Fe}$ (dashed line) is also shown. Note the very good agreement between model and observations. The largest differences appear during the earliest phase and the transition to the radioactive tail. At the earliest epochs, our bolometric corrections have the largest uncertainties because 

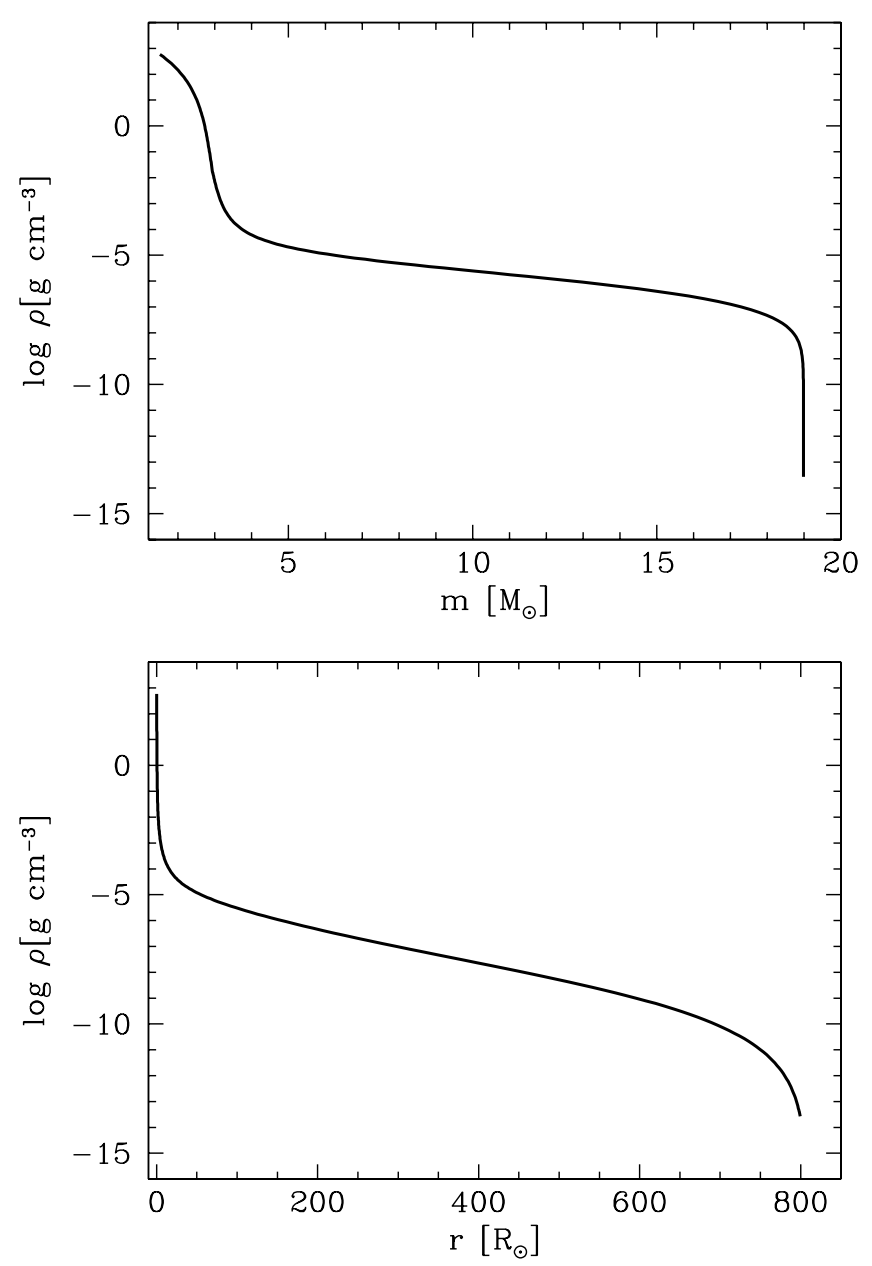

Figure 3. Initial density distribution with respect to interior mass (top) and radius (bottom) for the pre-SN model M19R8E1.25NI56.

during this time the UV flux-which is the main contribution of the luminosity - is not well constrained as noted in Bersten \& Hamuy (2009). During the transition between the plateau and the radioactive tail, the diffusion approximation breaks down because the object is almost completely recombined and the photosphere is not well defined. During the tail, the bolometric luminosity is completely determined by the luminosity of radioactive decay, which makes our calculations more reliable. Although not shown here, our model shows that the shape of the LC at the end of the plateau is very sensitive to the properties of the core, such as mixing and the form of the density transition between the helium-rich core and the hydrogen-rich envelope. On the other hand, the presence of an outer atmosphere can affect the shape of the LC at the earliest stages. During these epochs, and until the hydrogen recombination sets in, the LC samples the outermost layers as the photosphere is located far out in the object (see Section 3.1.3). So, even if it was possible to find a better fit to the observations by modifying the innermost or outermost structures, such efforts are rendered meaningless due to the uncertainties introduced by our approximations.

Figure 6 shows the photospheric-velocity evolution of our model compared with observed photospheric velocities as explained in Section 2.5. We have also included in the plot the spectroscopic velocities measured from the absorption minimum of the Fe II $\lambda 5169$ line (Jones et al. 2009). Note the very good overall agreement between model and observations. Fe II velocities match quite well the model photospheric velocities ex-

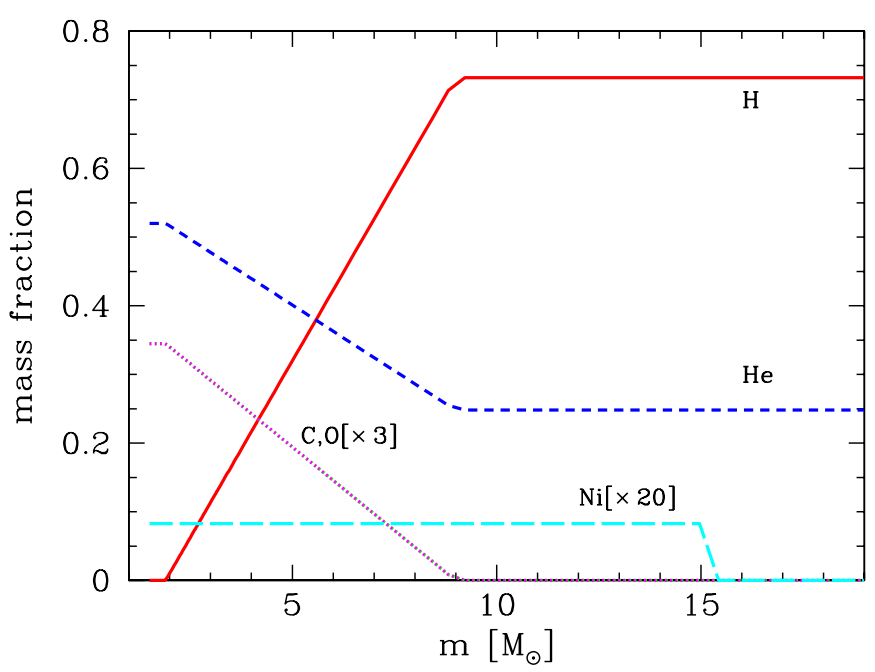

Figure 4. Abundance distribution in the pre-SN model M19R8E1.25NI56 with respect to Lagrangian mass. For clarity, the abundance of carbon $(\mathrm{C})$ and oxygen (O) was multiplied by 3 and the abundance of ${ }^{56} \mathrm{Ni}$ by 20 . Note that the ${ }^{56} \mathrm{Ni}$ is uniformly mixed in the outer envelope until $15 M_{\odot}$.

(A color version of this figure is available in the online journal.)

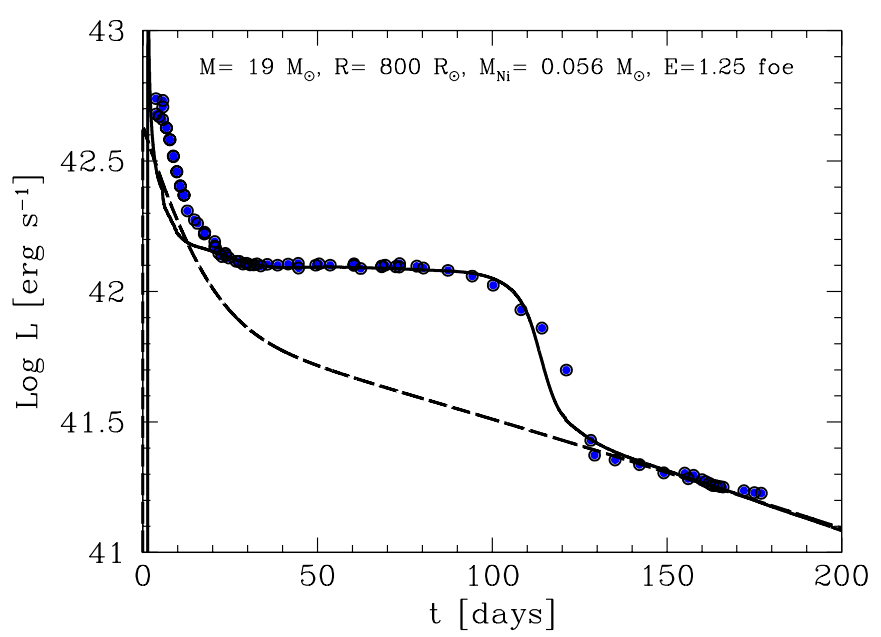

Figure 5. Bolometric LC for model M19R8E1.25NI56 (solid line) compared with the data of SN 1999em as calculated by Bersten \& Hamuy (2009; blue dots). The luminosity due to the ${ }^{56} \mathrm{Ni} \rightarrow{ }^{56} \mathrm{Co} \rightarrow{ }^{56} \mathrm{Fe}$ decay is also shown (dashed line). The physical parameters used in the model are indicated.

(A color version of this figure is available in the online journal.)

cept at the latest times. However, it is important to remark that the photospheric velocity is to be considered a good discriminator between models only at the early phases of the evolution while the object is not completely recombined. This is because, line velocities become poor photospheric velocity indicators with time, and the photosphere begins to lose its meaning in our models as the ejecta becomes nearly completely recombined at the end of the plateau.

As shown in Figures 5 and 6 we obtain a remarkably good agreement with observations (bolometric LC and photospheric velocity evolution) for SN $1999 \mathrm{em}$ despite the simplifications used in our code. Note that in spite of the mismatch between the model and observations during the early LC, the photospheric velocities, which are better determined than the $\mathrm{LC}$, show a good agreement at such epochs. These results are very encouraging and give us confidence in the ability of our code to infer physical parameters to study their effect on the observed quantities and ultimately to understand the physics of SNe II-P. 


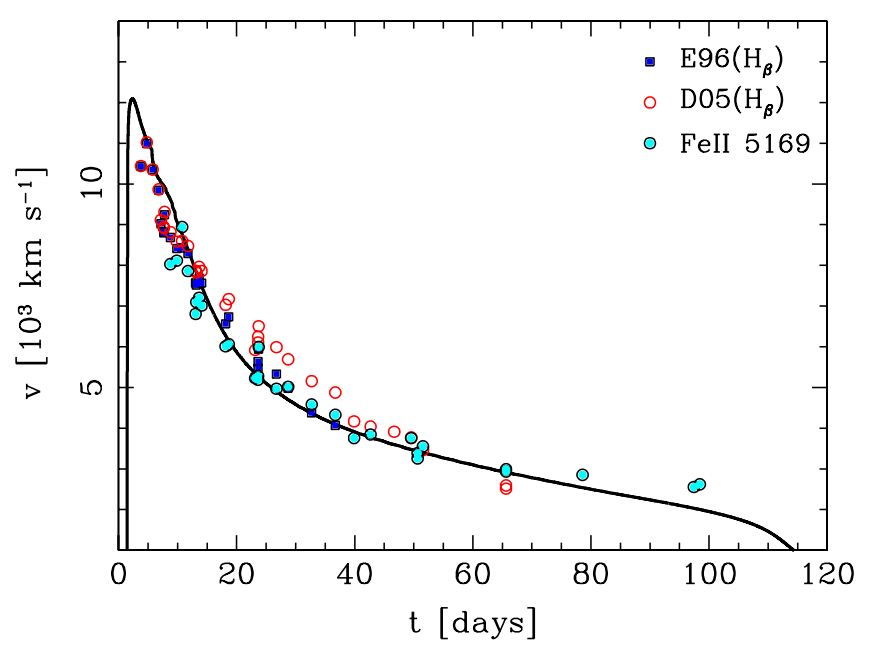

Figure 6. Evolution of the expansion velocity of the photosphere for model M19R8E1.25NI56 (solid line) compared with observed photospheric velocities calculated by Jones et al. (2009) using a calibration between the velocity derived from the absorption minimum of $\mathrm{H}_{\beta}$ and two atmosphere models: E96 (filled squares) and D05 (open circles). We also include the velocities calculated from Fe II $\lambda 5169$ line (filled circles) which are not transformed to photospheric velocity.

(A color version of this figure is available in the online journal.)

In the following subsections, we describe in some detail the evolution of the $\mathrm{SN}$ for this particular model. At a glance, the LC is distinguished by three phases: (1) an outburst followed by strong cooling, (2) a plateau, and (3) a radioactive tail. Each phase is essentially determined by the interplay between the main heating and cooling mechanisms. We thus focus our discussion on these processes along the $\mathrm{SN}$ evolution. As a reference, Table 1 gives a summary of some properties of the model at characteristic times during the evolution. Even though some uncertainties may arise on the detailed propagation of the SW due to the assumed mixing and energy injection, and also on the shock breakout because of the adopted radiative transport, we think that it is instructive to provide a description of the processes which occur during such phases. We emphasize that the details of the shock propagation do not directly affect the resulting $\mathrm{LC}$ and photospheric velocities, which we are mainly interested in modeling.

The initial phase of shock breakout is discussed in Section 3.1.1, the adiabatic-cooling phase where the homologous expansion is reached is addressed in Section 3.1.2, the cooling and recombination phase is described in Section 3.1.3, and finally the radioactive-decay processes that power the tail are explained in Section 3.1.4.

\subsubsection{Shock Wave Propagation and the Early Evolution}

A powerful SW begins to propagate outward through the envelope when we artificially inject energy near the center of the star (assumed to occur at $t=0$ ). This energy is initially released as internal energy, and part of it is rapidly transformed into kinetic energy (e.g., by $t=0.5$ days, the total energy is approximately equally divided between kinetic and internal energy). The velocities acquired by matter are so high that they exceed the local speed of sound, leading to the formation of a SW. The SW heats and accelerates the matter depositing mechanical and thermal energy into successive layers of the envelope until it reaches the surface, where photon diffusion dominates the energy transfer, and energy begins to be radiated away.
Figure 7 shows the effect of the SW propagation on different physical quantities (velocity, density, and temperature) inside the star. The shock front manifests as a sudden change in these quantities. As the shock moves outward, the material behind is accelerated and heated. Note that the outermost layer, with a sharp decline in density, acquires very high velocities. It represents a small fraction of the star. It is also clear from the velocity profiles that the inner parts of the object are decelerated. This deceleration is due to the interaction of the dense core with the extended hydrogen-rich envelope. The same figure also shows the changes in radius for different shells. From this we can deduce the location of the shock front at any time as that of the innermost shell, which has constant radius.

At $t=1.36$ days, the $\mathrm{SW}$ reaches the surface of the object, which produces the first electromagnetic manifestation of the explosion (although neutrinos and gravitational waves escape well before). The effective temperature and bolometric luminosity suddenly rise and reach their maximum values a few hours after breakout, specifically, at $t=1.47$ days with values of $L_{\text {peak }}=3 \times 10^{44} \mathrm{erg} \mathrm{s}^{-1}$ and $T_{\text {peak }}=1.1 \times 10^{5} \mathrm{~K}$. At these temperatures, the peak of the emitted spectrum is in the UV range. Also note that the color temperature is even higher than the effective temperature, as mentioned in Section 2.5.

Hereafter, the star begins to expand and cool very quickly, leading to an increase in photospheric radius and a decrease in temperature in the external layers. The bolometric luminosity abruptly decreases but, according to the decrease in effective temperature and the consequent shift of the emission peak to longer wavelengths, the luminosity in the optical range increases. As a result, a sharp peak in bolometric luminosity and temperature is produced, as shown in Figure 8. For example, in our model we obtain a decrease of 1.5 dex in luminosity only $6.8 \mathrm{hr}$ after peak brightness. During this time the total energy radiated is $2.2 \times 10^{48} \mathrm{erg}$, emitted essentially as a UV flash. The short duration of the breakout explains why so few SNe II-P have been observed during this phase.

At temperatures as high as those left by the passage of the $\mathrm{SW}$, the stellar matter is completely ionized, which implies that the breakout is accompanied by a strong increase in opacity. The position of the photosphere during the outburst nearly coincides with the outermost shell. This behavior continues until the onset of recombination. Therefore, at early stages prior to recombination, the velocity of matter in the photospheric position samples the very high velocities of the outermost layers and reaches values close to $1.2 \times 10^{4} \mathrm{~km} \mathrm{~s}^{-1}$.

From the energetics point of view, by $t=0.5$ days the total energy is approximately divided in equal proportions between internal, dominated by radiative contribution, and kinetic components. Short after breakout, the kinetic energy completely dominates the energetics (the energy radiated away at any given time is less than $1.5 \%$ of the total energy).

It is possible to estimate the average velocity during the SW propagation: given that the SW takes 1.36 days to emerge and considering a radius of $R=5.5 \times 10^{13} \mathrm{~cm}\left(800 R_{\odot}\right)$, we obtain an average speed of $v=4680 \mathrm{~km} \mathrm{~s}^{-1}$. It is also interesting to calculate the expected time for breakout using the analytic expression given by Shigeyama et al. (1987):

$t_{\mathrm{bk}} \simeq 1.6\left(\frac{R_{0}}{50 R_{\odot}}\right) \times\left[\left(\frac{M_{\mathrm{ej}}}{10 M_{\odot}}\right) /\left(\frac{E}{1 \times 10^{51} \mathrm{erg}}\right)\right]^{1 / 2} \mathrm{hr}$,

where $R_{0}$ is the initial radius, $M_{\mathrm{ej}}$ is the ejected mass, and $E$ is the explosion energy. Using the values for our initial model we 

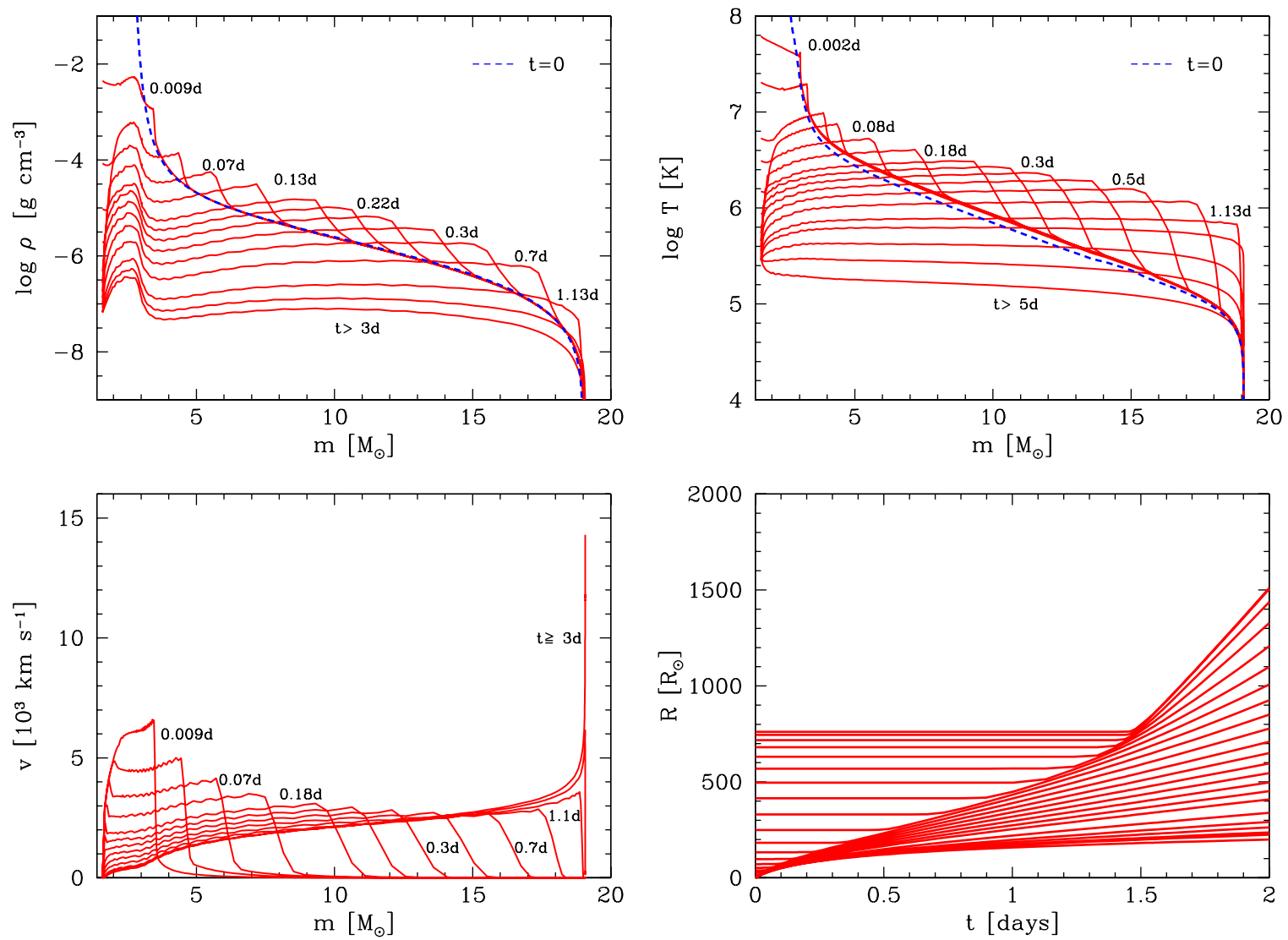

Figure 7. Changes in density (top left), temperature (top right), and velocity (bottom left) profiles as a function of interior mass during shock propagation for model M19R8E1.25NI56. Some of the curves are labeled with the time elapsed since the energy is injected. The initial density and temperature profiles are also shown $(t=0$; dashed line). Note that a very small amount of material near the surface is strongly accelerated as the SW passes through the steep density gradients present in the outermost layers. Bottom right: temporal evolution of the radial coordinates corresponding to different mass shells in the interior.

(A color version of this figure is available in the online journal.)
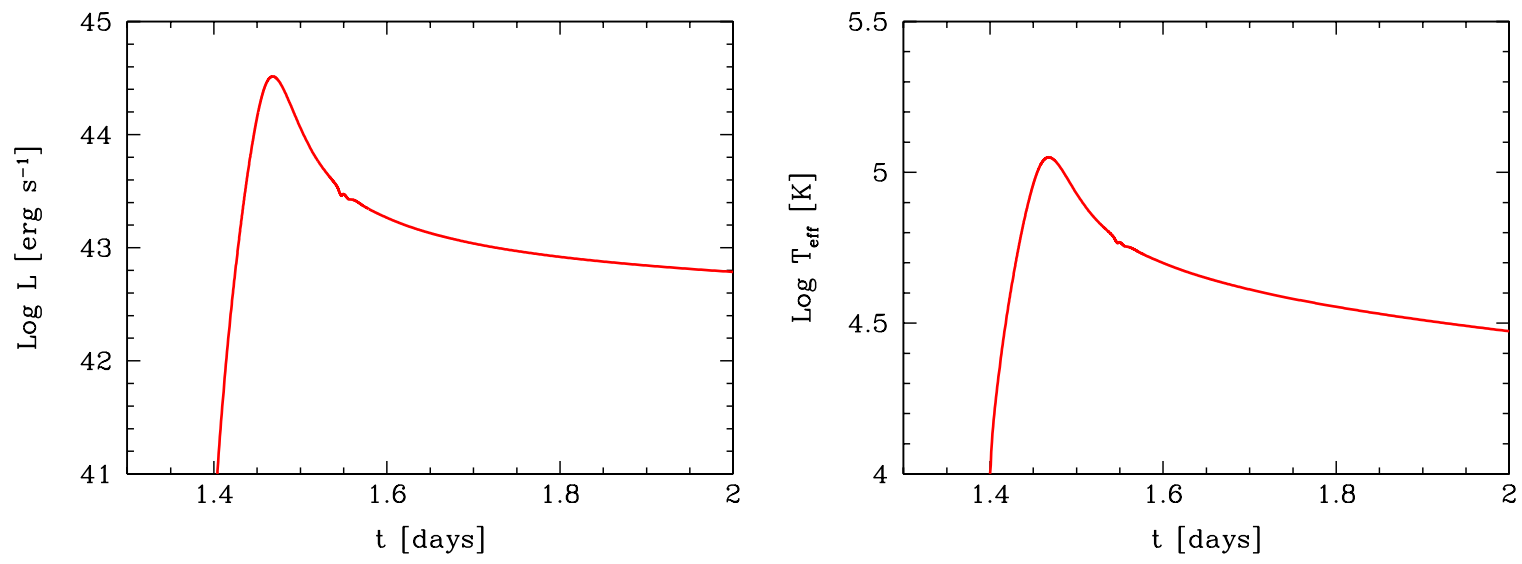

Figure 8. Bolometric luminosity (left) and effective temperature (right) during shock breakout.

(A color version of this figure is available in the online journal.)

Table 1

Properties of Model M19R8E1.25NI56 at Selected Time of Their Evolution

\begin{tabular}{lcccccccc}
\hline \hline Phase & $\begin{array}{c}t \\
(\text { days })\end{array}$ & $\begin{array}{c}\log \left(L_{\mathrm{bol}}\right) \\
\left(\mathrm{erg} \mathrm{s}^{-1}\right)\end{array}$ & $\begin{array}{c}\log \left(T_{\text {eff }}\right) \\
(\mathrm{K})\end{array}$ & $\begin{array}{c}\log \left(T_{\mathrm{ph}}\right) \\
(\mathrm{K})\end{array}$ & $\begin{array}{c}R_{\mathrm{ph}} \\
\left(10^{14} \mathrm{~cm}\right)\end{array}$ & $\begin{array}{c}v_{\mathrm{ph}} \\
\left(10^{8} \mathrm{~cm} \mathrm{~s}^{-1}\right)\end{array}$ & $\begin{array}{c}E_{\mathrm{rad}} / E_{0}{ }^{\mathrm{a}} \\
(\%)\end{array}$ & $\begin{array}{c}E_{\mathrm{K}} / E_{0}{ }^{\mathrm{a}} \\
(\%)\end{array}$ \\
\hline Peak & 1.5 & 44.5 & 5.05 & 5.03 & 0.54 & 3.36 & 0.04 & 72 \\
Adiabatic cooling & 5 & 42.4 & 4.10 & 4.05 & 3.8 & 11.5 & 0.2 & 93 \\
Plateau & 50 & 42.1 & 3.73 & 3.77 & 14.7 & 3.47 & 0.65 & 99 \\
Transition & 114 & 41.7 & 3.74 & 3.74 & 10 & 1 & 1.2 & 99.7 \\
\hline
\end{tabular}

Note. ${ }^{\text {a }} E_{\mathrm{rad}}$ is defined by $\int_{0}^{t} L_{\mathrm{bol}} d t$ and $E_{K}$ is the total kinetic energy in the mass motions. In the table, we show these quantities normalized to the initial injected energy of model M19R8E1.25NI56, $E_{0}=1.25$ foe. 
obtain $t_{\mathrm{bk}}=1.26$ days, in good agreement with our numerical calculation.

\subsubsection{Adiabatic Cooling and Homologous Expansion}

The breakout is followed by a violent expansion, resulting in the cooling of the outermost layers. During expansion, only a small fraction of the photon energy can diffuse into the surroundings. Therefore, it is possible to consider the cooling process to be approximately adiabatic and this approximation remains valid while the timescale for radiation diffusion is much longer than the expansion timescale. ${ }^{7}$ Note that there are two mechanisms to cool an SN: (1) loss of photons or diffusion cooling and (2) its own expansion or "adiabatic cooling." If one of these processes dominates we say that the cooling is carried out by that process. In our model, more than $90 \%$ of the decrease of internal energy is due to adiabatic cooling up to 18 days after the explosion, when the first layers with neutral hydrogen appear. After that, diffusion cooling begins to be significant and the adiabatic cooling phase comes to an end.

The internal temperature (and internal energy) decreases almost adiabatically, i.e., proportional to $r^{-1}$ due to the dominance of the radiative term, and quickly reaches a value near the recombination temperature of hydrogen. Quantitatively, the effective temperature goes from values close to $10^{5} \mathrm{~K}$ at the time of the burst when the matter is totally ionized to $T_{\text {eff }}=10^{4} \mathrm{~K}$ five days later. At these temperatures hydrogen begins to recombine. At the same time, the luminosity reaches $L=1.9 \times 10^{42} \mathrm{erg} \mathrm{s}^{-1}$ and the photospheric radius rapidly increases to $R_{\mathrm{ph}}=5.2 \times 10^{14}$ $\mathrm{cm}$. After that, the luminosity decreases slightly as a result of the slower decrease in temperature and continuous increase in radius. By day 18 , when the first layers of neutral hydrogen appear, we have $T_{\text {eff }}=6960 \mathrm{~K}, L=1.2 \times 10^{42} \mathrm{erg} \mathrm{s}^{-1}$, and $R_{\mathrm{ph}}=9.1 \times 10^{14} \mathrm{~cm}$. Note the slight change in luminosity, of only 0.14 dex, between day 7 and 18 .

A few days after breakout, the acceleration of the material comes to an end and the expansion becomes homologous. The matter reaches velocities of the order of $1.2 \times 10^{4} \mathrm{~km} \mathrm{~s}^{-1}$ in the outermost layers and the object enters a state of free expansion where forces of pressure and gravitation do not have any dynamical effect on the system. The homologous regime is characterized by a constant velocity in each layer, a linear growth of the radial coordinate with time $(r \propto t)$, and a density distribution decreasing with time as $\rho \propto t^{-3}$. This behavior is clearly shown in Figure 7 for $t>3$ days and in Figure 9. The nearly constant shape of the density and temperature profiles at late times are a result of the expansion being approximately homologous. This is also evident in the linear behavior of the radial coordinates for different mass shells. The condition of constant velocity is very nearly satisfied for each shell, although the transition between acceleration and homologous expansion happens at slightly different times for different mass shells (see Figure 9). ${ }^{8}$

As mentioned in Section 3.1.1, at the time of breakout the internal energy of the envelope is small compared to the kinetic energy. Moreover, the object expands by a factor of $\sim 17$ to a radius of $R_{\mathrm{ph}} \sim 9 \times 10^{14} \mathrm{~cm}$, before it becomes transparent.

\footnotetext{
7 The expansion timescale, $\tau_{\mathrm{h}}=R / v$, increases with time while the diffusion timescale, $\tau_{\mathrm{d}}=\kappa \rho R^{2} / c$, decreases because $\rho \propto R^{-3}$. Thus, there is a time after which the condition for this approximation breaks down.

8 After day 8, the photosphere remains located in shells where homology has been reached. Therefore, our models show that the EPM, which is used to estimate distances assuming homologous expansion, must be applied at epochs later than this.
}

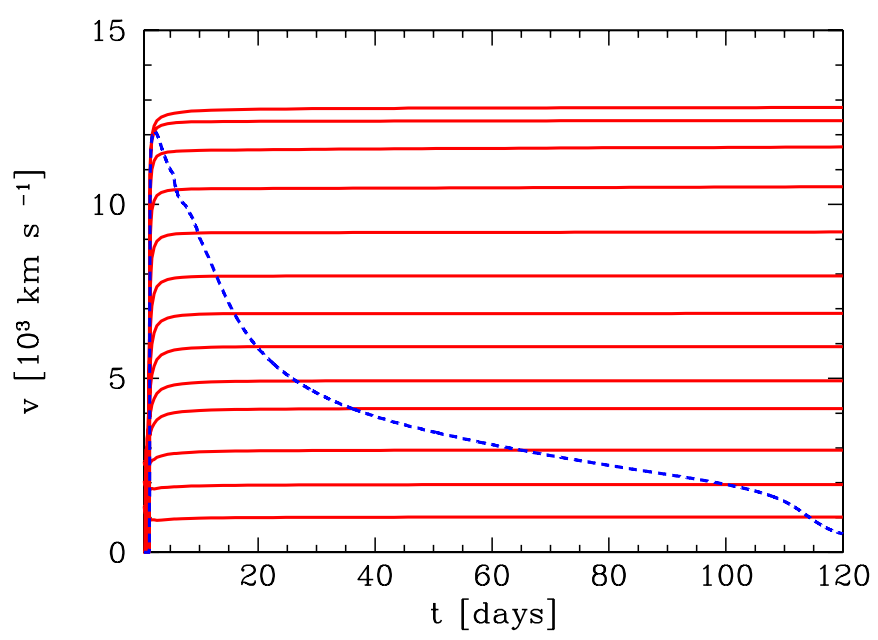

Figure 9. Evolution of the velocity for different layers (solid lines) and the velocity of material at the photospheric position (dashed line). Note that the asymptotic constant velocity is reached at a different time for each shell.

(A color version of this figure is available in the online journal.)

Thus, most of the internal energy left by the shock passage is degraded by the adiabatic expansion, and only a small residual will be released as observable radiation in the following phase.

\subsubsection{Cooling and Recombination Wave}

The appearance of regions with neutral hydrogen determines the onset of an RW. The duration of the RW is related to the total hydrogen mass and how deep hydrogen has been mixed in the initial model. As time goes on, hydrogen recombination occurs at different layers of the object as a wave propagates inward (in Lagrangian coordinate; see Figure 10, top left). Because the opacity is dominated by electron scattering, it strongly decreases outward of the recombination front (Figure 10, top right), increasing the transparency of these layers and allowing the radiation to easily go away. Consequently, the internal energy (mostly of the radiation field) is efficiently radiated away, and the temperature drops sharply from $\sim 10,000 \mathrm{~K}$ to $\sim 5500 \mathrm{~K}$ at the recombination front (Figure 10, bottom left). In other words, a cooling wave associated with the transparency and induced by an RW propagates through the envelope. This is usually called "cooling and recombination wave" (CRW).

In our model, the recombination begins approximately at day 18. After that, a CRW develops which moves inward in mass until all the matter is completely recombined by day 120 when the recombination front arrives at the innermost layers of the H-rich envelope (at $m=2 M_{\odot}$ for our model), and the luminosity suddenly drops, defining the end of this phase. The propagation of the CRW is clear from a glance at Figure 10, where the evolution of the fraction of ionized hydrogen and temperature profiles as a function of mass for selected times are shown. Note also the behavior of the opacity (see Figure 10, top right), which depends strongly on the ionization state of the matter. In our model, a strong drop in the opacity of the outer layers is seen at day $\sim 18$, which leads to a considerable decrease in the optical depth of these layers and an inward drift in mass of the photosphere-defined at a fixed optical depth of $\tau=2 / 3$. The photosphere begins to follow the CRW, as shown in Figure 10 where the points indicate the photospheric position. It is important to note that we define the position of the photosphere using only continuum opacity sources, i.e., excluding the opacity floor (see Section 2.5). 

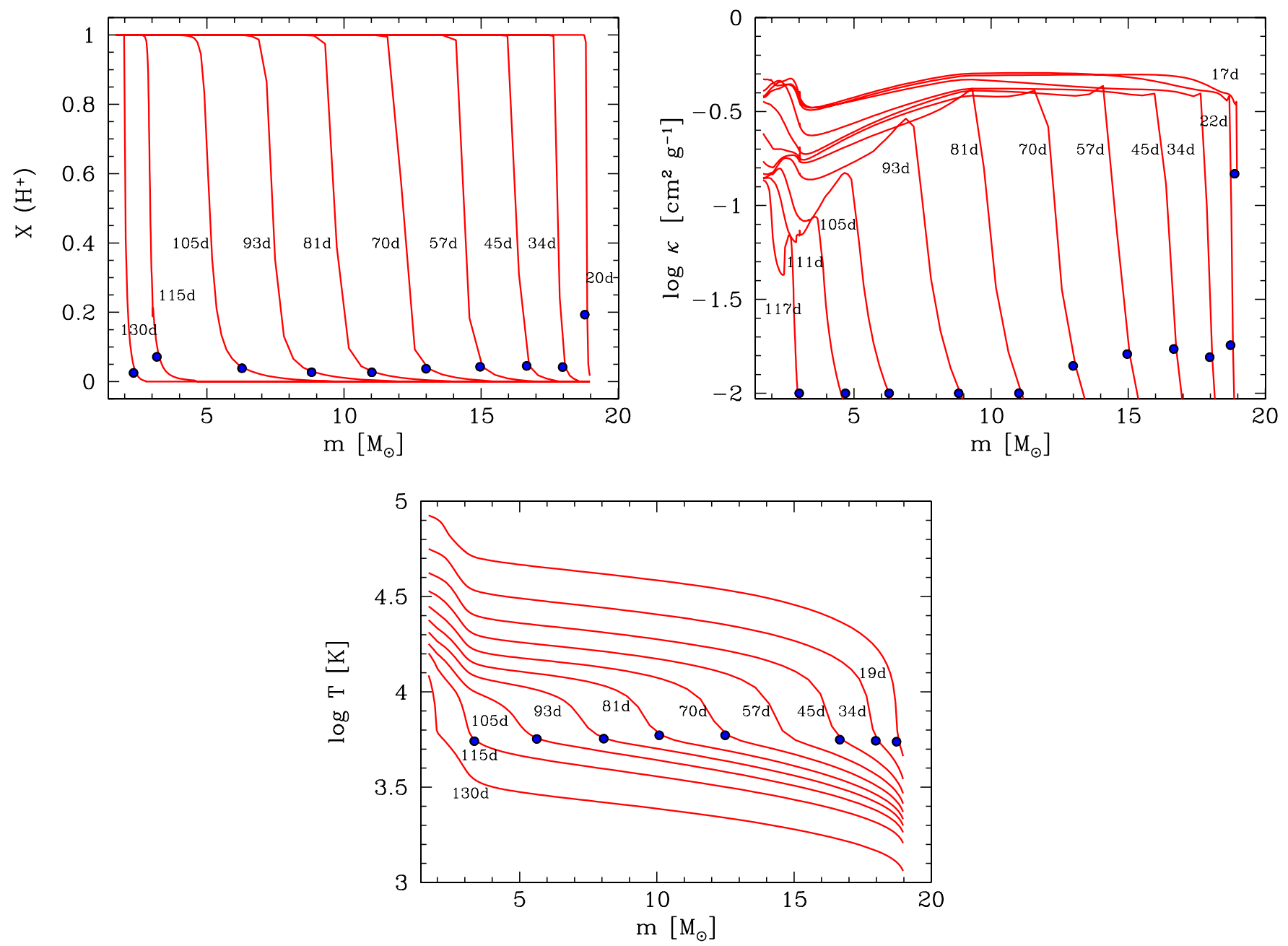

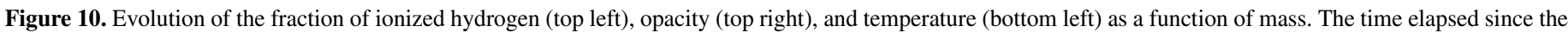

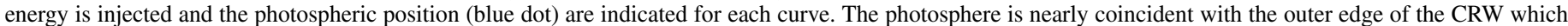

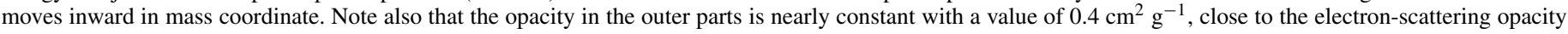
for matter composed by pure hydrogen, above which it suddenly drops.

(A color version of this figure is available in the online journal.)

The photosphere, as defined here, is nearly coincident with the outer edge of the CRW. Therefore, it is just the location of the photosphere with respect to the CRW what sets the value of the photospheric temperature close to the temperature of hydrogen recombination $(T \sim 5500 \mathrm{~K})$. However, note that the effective temperature does not necessarily have a constant value. Since the effective temperature is defined as $T_{\mathrm{eff}}^{4}=L / \pi \sigma R_{\mathrm{ph}}^{2}$ and the luminosity remains nearly constant outside the recombination front (see Figure 11), any changes in effective temperature are related to changes in photospheric radius.

The CRW divides the object into two distinct regions: (1) an inner zone which is hot, optically thick, and ionized and (2) an outer zone which is relatively cold, optically thin, and completely recombined. Matter in the inner zone is opaque: radiative transfer is too inefficient to produce any appreciable flow of energy (this would be strictly fulfilled if ${ }^{56} \mathrm{Ni}$ were confined to the innermost layers; see the discussion) and the matter cools down almost adiabatically. On the other hand, the external layers are transparent and practically do not radiate. Therefore, it is within the CRW where almost the entire radiant flux is released. When matter passes through the CRW, particles begin to be cooled by radiation, emitting more light than they absorb, and the radiant flux increases. This way, the radiative flux emerging from the CRW front carries away internal energy of the matter that is cooled by the wave. More details on the properties of the CRW are given by Grassberg et al. (1971).

Note that the bulk of the radiation does not diffuse to the photosphere; the photosphere instead moves inward, allowing the radiation to escape sooner than it would for a photosphere fixed at the outer boundary of the ejected mass. That is, the recombination process is responsible for the energy release during this phase. It should be noted that most of this energy comes from the energy deposited by the SW and not from the recombination itself. In order to test the previous statement, we ran our code without including the energy released by recombination of ions with electrons. No appreciable change was found; quantitatively, the differences between both calculations are less than 0.04 dex during all the evolution. We have included a figure in the online edition (Figure 17) showing this comparison.

Note, however, that in model M19R8E1.25NI56 where we assumed an extended ${ }^{56} \mathrm{Ni}$ mixing, the flux of energy inside the CRW is not negligible (see the left panel of Figure 11). This is due to the fact that the photosphere meets regions with radioactive material earlier than in the case where ${ }^{56} \mathrm{Ni}$ is confined to the innermost layers. Therefore, the energy deposited by radioactive decay provides additional power for 

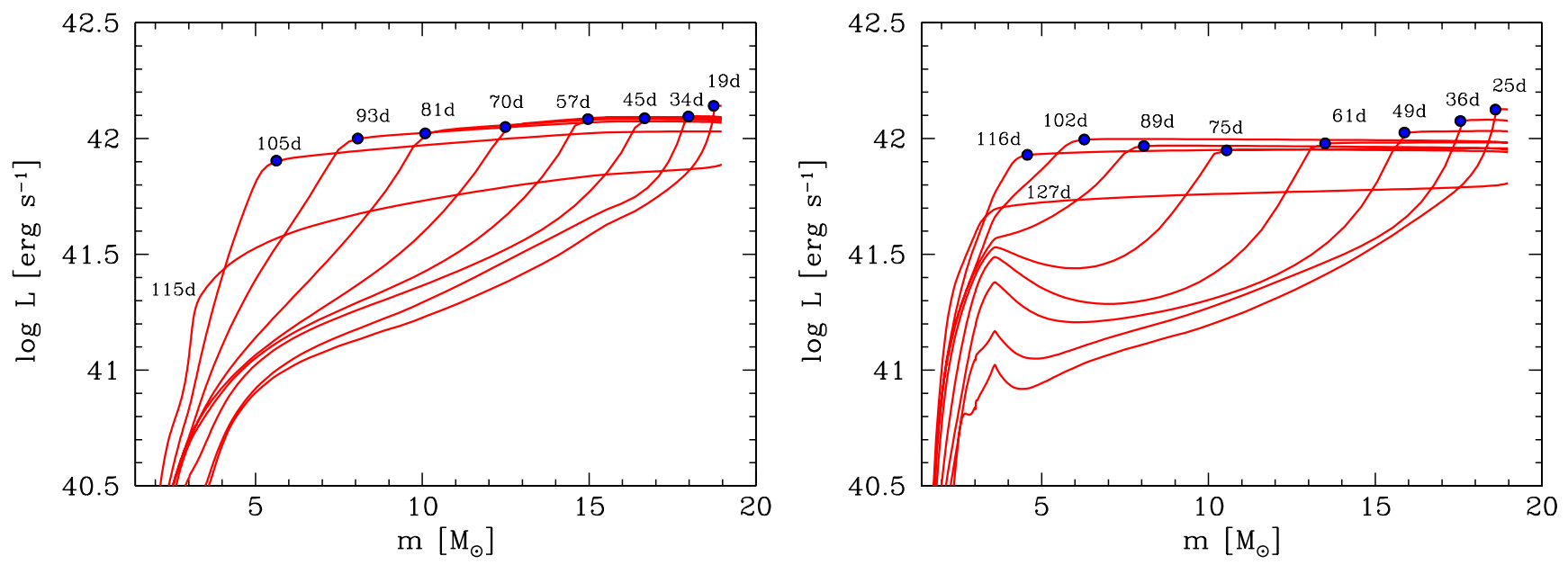

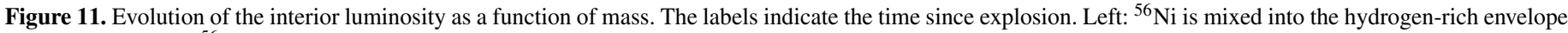

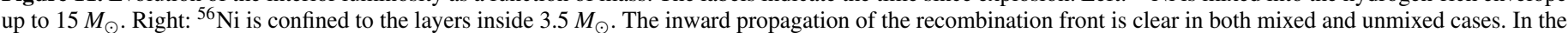

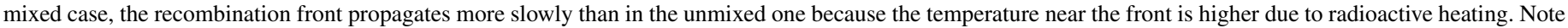
that for the unmixed case the outward diffusion of the radioactive energy is clear, while for the mixed case this effect occurs too early to be noticeable.

(A color version of this figure is available in the online journal.)

the LC. A different behavior is seen when ${ }^{56} \mathrm{Ni}$ is confined to the innermost layers (inside $2.5 M_{\odot}$; see the right panel of Figure 11). In this case, the statement that the energy flux inside the CRW is very small is fulfilled at least until day $\sim 110$ where the radiative diffusion of the radioactive decay from the central layers begins to dominate. At earlier times, it is possible to see the outward diffusion of radioactive energy. Note also that for both models the luminosity outside the front is nearly constant.

The assumption of extended mixing was necessary in order to obtain a plateau as flat as the one observed for SN 1999em. Figure 12 shows a comparison of the bolometric LC for three cases: (1) with extended ${ }^{56} \mathrm{Ni}$ mixing, (2) no mixing, and (3) without ${ }^{56} \mathrm{Ni}$. For case (1), ${ }^{56} \mathrm{Ni}$ begins to affect the LC by day $\sim 35$ while for case (2) the effect of nickel heating is delayed until day $\sim 75$. On the other hand, in the former case there is a less direct energy deposition at the center of the object, and the plateau declines earlier and steeper to the tail than in case (2). Our assumption of mixing of ${ }^{56} \mathrm{Ni}$ into the hydrogen envelope is not unreasonable as shown in studies of SN 1987A (Shigeyama et al. 1988; Woosley et al. 1988; Arnett 1988; Blinnikov et al. 2000, among others). However, it is important to mention that Utrobin (2007) found an excellent agreement with observations of SN $1999 \mathrm{em}$ by confining ${ }^{56} \mathrm{Ni}$ to the innermost layers and good agreement with observations of SN 1987A assuming moderate ${ }^{56} \mathrm{Ni}$ mixing (Utrobin 1993, 2004).

The evolution of the velocity of matter at the photospheric position $v_{\mathrm{ph}}$, the photospheric radius $\left(R_{\mathrm{ph}}\right)$, and the mass above the photosphere $\left(M_{\mathrm{ph}}\right)$ are shown in Figure 13. Initially, the photospheric velocity evolves rapidly, the photospheric position follows a linear behavior, and there is very little mass above the photosphere. Later on, when the recombination sets in, $R_{\mathrm{ph}}$ begins to differ noticeably from the linear behavior, the mass above the photosphere increases, and $v_{\mathrm{ph}}$ decreases because it samples increasingly inner, slower material. Finally, when all the matter is recombined, $R_{\mathrm{ph}}$ and $v_{\mathrm{ph}}$ sharply turn down and the luminosity undergoes a rapid decrease. Note, however, that the photospheric radius does not drop immediately. This is due to the heating caused by radioactive decay which produces some ionization of the gas. On the other hand, the luminosity undergoes a rapid decrease to values close to the luminosity of radioactive decays. If there was no ${ }^{56} \mathrm{Ni}$ (case 3), the $\mathrm{SN}$

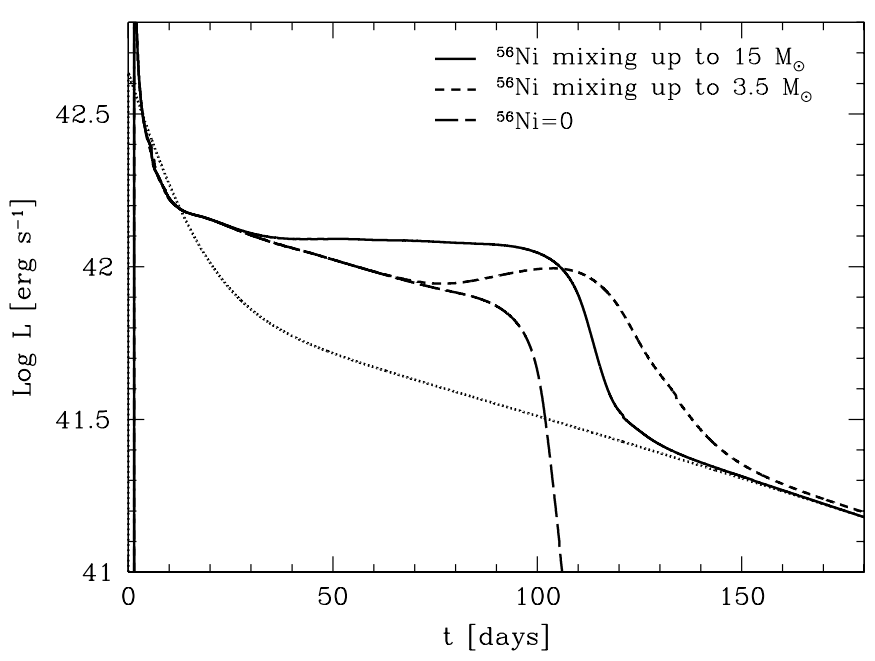

Figure 12. Comparison between bolometric LCs for extended ${ }^{56} \mathrm{Ni}$ mixing (solid line) and a model with mixing of ${ }^{56} \mathrm{Ni}$ up to $3.5 M_{\odot}$ (short-dashed line). We also include the case of a model without ${ }^{56} \mathrm{Ni}$ (long-dashed line) in which case LC falls abruptly after the plateau phase. The presence of ${ }^{56} \mathrm{Ni}$ extends the plateau and increases the luminosity. This is essentially produced when the CRW reaches layers with ${ }^{56} \mathrm{Ni}$ which can thereby power the LC directly. The extended ${ }^{56} \mathrm{Ni}$ mixing reveals this effect earlier on the evolution, which produces a flat plateau in concordance with the observations of SN 1999em.

luminosity would abruptly vanish at this point, as shown with a long-dashed line in Figure 12.

In conclusion, the CRW has a duration of $\sim 100$ days. The luminosity experiences a small change during the CRW propagation. In order to produce a plateau as flat as that observed for SN 1999em, we need to invoke Ni mixing in the H-rich envelope. Thus, the plateau can be seen as a combination of CRW properties plus some additional energy provided by radioactivity. Defining, from an empirical point of view, the plateau phase as the period of time when the luminosity remains constant within $0.5 \mathrm{mag}$ of the value at day 50 , its duration is 103.5 days, and the total energy emitted during this phase is $\sim 1.12 \times 10^{49} \mathrm{erg}$. Note that with such a definition the plateau phase includes the final stages of the adiabatic cooling phase as well. 


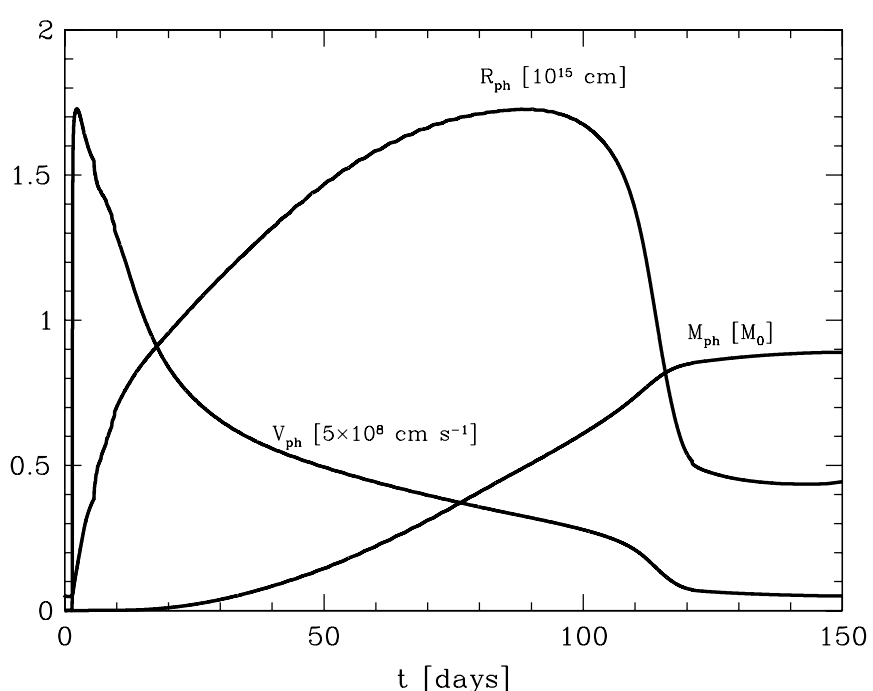

Figure 13. Evolution of the photospheric radius $\left(R_{\mathrm{ph}}\right)$ in units of $10^{15} \mathrm{~cm}$, the photospheric velocity $\left(V_{\mathrm{ph}}\right)$ in units of $5 \times 10^{8} \mathrm{~cm} \mathrm{~s}^{-1}$, and the mass above the photosphere $\left(M_{\mathrm{ph}}\right)$ in units of $M_{0}\left(M_{0}=19 M_{\odot}\right)$ for the model M19R8E1.25NI56.

\subsubsection{Radioactive Tail}

The late behavior of the LC (at $t>130$ days) is dominated by the energy released from radioactive decay. Without radioactive material, the luminosity would abruptly vanish when hydrogen gets completely recombined as shown in Figure 12. Instead, the observed LC decreases to values close to the instantaneous rate of energy deposition by the radioactive decay:

$$
L \sim 1.43 \times 10^{43} M_{\mathrm{Ni}} / M_{\odot} \exp (-t / 111.3) .
$$

The decline of the LC at $t>130$ days is nearly linear, in concordance with the exponential decline of the radioactive decay law. That is, the diffusion time for optical photons becomes small enough to radiate away the decay energy instantaneously, while the gamma-ray optical depth is still sufficiently large in order to allow a nearly complete local deposition of the decay energy. The bolometric luminosity in this part of the LC is a direct measure of the Ni mass synthesized in the explosion.

\subsection{Comparison with Other Hydrodynamical Models of SN 1999em}

In this section, we compare our results with those of previous hydrodynamical studies of SN 1999em. As mentioned in Section 3, there are two previous hydrodynamical studies of SN 1999em: one given by Baklanov et al. (2005, BBP05, hereafter) and another by Utrobin (2007, U07, hereafter). In Table 2, we summarize the physical parameters obtained for
SN 1999em in the three studies along with the distance, explosion time, and the chemical composition assumed in each model. It is interesting to note that the three studies employ initial density distributions and chemical composition distributions of non-evolutionary models. In our work, we assume $\mathrm{H}$ and $\mathrm{He}$ profiles very close to those adopted by U07, yet a very different ${ }^{56} \mathrm{Ni}$ distribution. While we assume an extended, uniform mixing of Ni (see Figure 4), U07 confined ${ }^{56} \mathrm{Ni}$ to the innermost layers (see their Figure 2). In the case of BBP05, $\mathrm{H}$ and $\mathrm{He}$ were assumed to be uniformly mixed throughout the envelope and a radial distribution of ${ }^{56} \mathrm{Ni}$ up to $\sim 15 M_{\odot}$ was adopted (see their Figure 9). Therefore, our ${ }^{56} \mathrm{Ni}$ distribution and the resulting ${ }^{56} \mathrm{Ni}$ mass are in better agreement with those of BBP05 than those of U07. We also note that the mass of the compact remnantwhich is left out of the calculations-is different in all three works. We assume a compact remnant of $1.4 M_{\odot}$, while U07 assumed $1.58 M_{\odot}$ and BBP05 removed everything within a radius of $R_{C}=0.1 R_{\odot}$.

The approaches used in each work are very different. U07 used a hydrodynamical code with a one-group approximation for the radiative transport, including non-LTE treatment of opacities and thermal emissivity, non-thermal ionization, and expansion opacity. Their calculations, although involving a more sophisticated radiative transfer treatment, yielded bolometric LCs as in our code. However, the procedure to produce the observed bolometric LC for SN 1999em was quite different in both cases. While U07 based their calculations on the integration of $U B V R I$ photometry with a constant value of 0.19 dex added to take into account the infrared luminosity, we derived UBVRIJKLM bolometric luminosities from color-dependent bolometric corrections. As noted by Bersten \& Hamuy (2009), the inclusion of the $L$ and $M$ bands in the calculation of the bolometric correction during the tail phase is very important and it most likely leads to the difference in the nickel mass estimated in this work and in U07. BBP05, in turn, used a multi-group hydrodynamical code which allows the calculation of LC in different photometric bands. This code also included the expansion opacity effect. Therefore, BBP05 were able to compare their model with $U B V R I$ LC separately without being affected by the uncertainties in the calculation of bolometric luminosities.

As shown in Table 2, the parameters yielded by our calculations are intermediate between those estimated by BBP05 and U07. The largest differences are $2.6 M_{\odot}$ in mass, $500 R_{\odot}$ in radius, 0.3 foes in energy, and $0.045 M_{\odot}$ in ${ }^{56} \mathrm{Ni}$ mass. It is quite satisfactory that our simple prescription for the radiative transfer yields physical parameters similar to those obtained from more sophisticated codes. However, it should be taken into account that (1) the models and methods used in each study are quite different, (2) there are differences in assumed quantities, such as distance, explosion time, mass cut, photometry, and (3) there

Table 2

Comparison between Physical Parameters for SN 1999em from Three Different Hydrodynamical Codes

\begin{tabular}{lccccccccc}
\hline \hline Code & $\begin{array}{c}D \\
(\mathrm{Mpc})\end{array}$ & $\begin{array}{c}t_{0} \\
(\mathrm{JD}-2451000)\end{array}$ & $X_{\text {sup }}$ & $Z$ & $\begin{array}{c}E \\
(\text { foe })\end{array}$ & $\begin{array}{c}M \\
\left(M_{\odot}\right)\end{array}$ & $\begin{array}{c}R \\
\left(R_{\odot}\right)\end{array}$ & $\begin{array}{c}M_{\text {Ni }} \\
\left(M_{\odot}\right)\end{array}$ & $\begin{array}{c}\text { Ni Mixing } \\
\left(M_{\odot}\right)\end{array}$ \\
\hline This work & 11.7 & 477.90 & 0.735 & 0.02 & 1.25 & 19 & 800 & 0.056 & $\sim 15$ \\
BBP05 $^{\text {a }}$ & 12 & 468.90 & 0.7 & 0.004 & 1 & 18 & 1000 & 0.06 & $\sim 15$ \\
U07 $^{\mathrm{b}}$ & 11.7 & 476.90 & 0.735 & 0.017 & $1.3 \pm 0.1$ & $20.58 \pm 1.2$ & $500 \pm 200$ & $0.036 \pm 0.009$ & $\sim 2.5$ \\
\hline
\end{tabular}

Notes.

${ }^{\text {a }}$ Baklanov et al. (2005).

${ }^{\mathrm{b}}$ Utrobin (2007). 
Table 3

Model Parameters

\begin{tabular}{lcccc}
\hline \hline Model & $\begin{array}{c}\text { Mass } \\
\left(M_{\odot}\right)\end{array}$ & $\begin{array}{c}\text { Radius } \\
\left(R_{\odot}\right)\end{array}$ & $\begin{array}{c}\text { Energy } \\
(\text { foe })\end{array}$ & $\begin{array}{c}\text { Ni } \\
\text { Mixing }\end{array}$ \\
\hline M12R8E1NI56 & 12 & 800 & 1 & 0.8 \\
M12R8E08NI56 & 12 & 800 & 0.8 & 0.8 \\
M12R8E05NI56 & 12 & 800 & 0.5 & 0.8 \\
M12R5E1NI56 & 12 & 500 & 1 & 0.8 \\
M12R10E1NI56 & 12 & 1000 & 1 & 0.8 \\
M12R8E1NI56M & 12 & 800 & 1 & 0.5 \\
M12R8E1NI56IN & 12 & 800 & 1 & 0.2 \\
M12R15E05NI56 & 12 & 1500 & 0.5 & 0.8 \\
M12R15E08NI56 & 12 & 1500 & 0.8 & 0.8 \\
M14R8E1NI56 & 14 & 800 & 1 & 0.8 \\
M14R10E08NI56 & 14 & 1000 & 0.8 & 0.8 \\
M14R12E08NI56 & 14 & 1200 & 0.8 & 0.8 \\
M14R8E1NI56M & 14 & 800 & 1 & 0.5 \\
M14R8E1NI56I & 14 & 800 & 1 & 0.2 \\
M14R8E1.1NI56I & 14 & 800 & 1.1 & 0.2 \\
M14R10E09NI56M & 14 & 1000 & 0.9 & 0.5 \\
M14R10E09NI56I & 14 & 1000 & 0.9 & 0.2 \\
M14R12E09NI56M & 14 & 1200 & 0.9 & 0.5 \\
M14R12E09NI56I & 14 & 1200 & 0.9 & 0.2 \\
\hline
\end{tabular}

Note. ${ }^{a}$ The degree of ${ }^{56} \mathrm{Ni}$ mixing is given as a fraction of the initial mass of the model $\left(M_{0}\right)$.

may be a degree of degeneracy among explosion energy, initial radius, and initial mass within each model. Therefore, the present comparison does not help to provide a clear assessment of the validity of our physical assumptions and of the predictive power of our model.

\section{LOW-MASS SN 1999em}

The mass of the progenitors of SNe II-P can be derived from hydrodynamical modeling of LCs and expansion velocities, or from the detection of the pre-SN object in archival images of the host galaxy in connection with stellar evolution models. At the moment, three progenitor stars of SNe II-P have been firmly detected and there have been negative detections for 17 other objects which have led to upper limits of the progenitor star masses (Smartt et al. 2009). Among these, only three SNe II-P have masses derived using hydrodynamical models: SN 1999em, SN 2004et, and SN 2005cs. In all of these cases, the masses estimated from the hydrodynamical modeling are systematically higher than the values derived from the other method. These discrepancies have been noted previously in the literature (Utrobin \& Chugai 2008; Smartt 2009). For the particular case of SN $1999 \mathrm{em}$, the pre-SN images give an upper limit of $15 M_{\odot}$ for the progenitor star in the ZAMS. It is expected that the mass of the pre-SN object should be lower than this due to possible mass-loss episodes during the evolution of the star. In this section, we use our hydrodynamical model to explore a lowmass range, consistent with pre-SN imaging of SN 1999em, in order to test how well we can reproduce the observed properties of this object. Although the adoption of non-evolutionary initial models may introduce an uncertainty in the actual progenitor mass (see Section 2.4), it is interesting to study low- and highmass models for this $\mathrm{SN}$ in a comparative way.

We calculate several models using two different values of the pre-SN mass: $M=12 M_{\odot}$ (see Figures 14 and 15), and $M=14 M_{\odot}$ (see Figures 16 and 17). In all figures, we show the bolometric LCs and photospheric velocities $\left(v_{\mathrm{ph}}\right)$ for different values of the injected energy $(E)$, initial radius $(R)$, and degree of

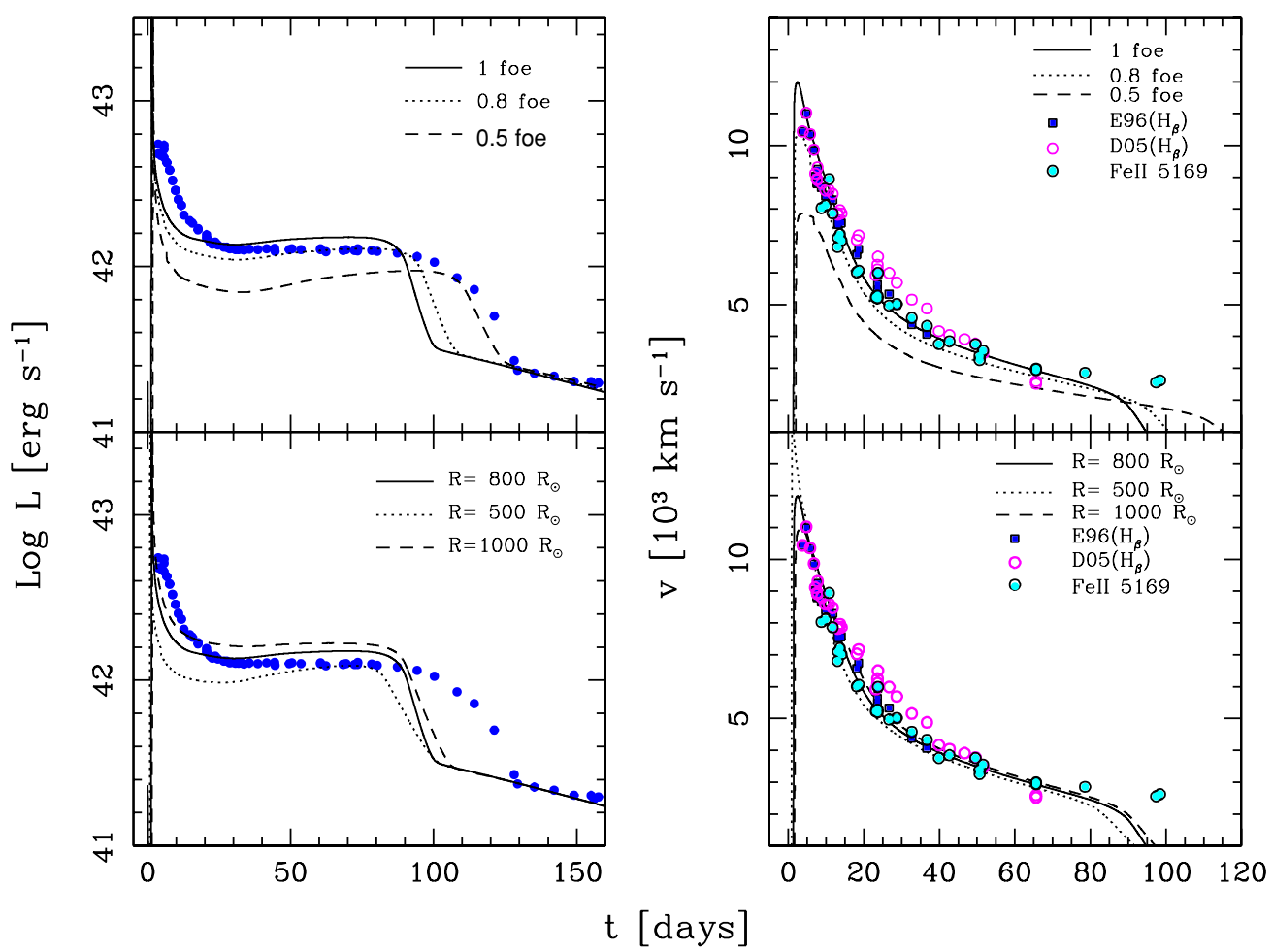

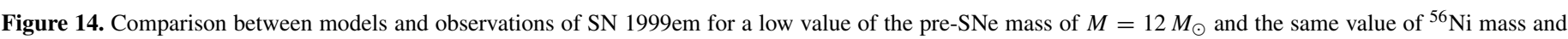

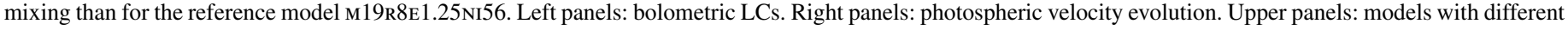

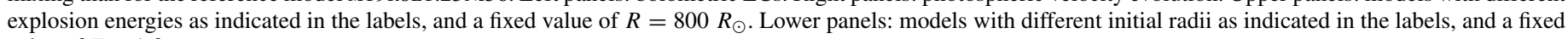
value of $E=1$ foe.

(A color version of this figure is available in the online journal.) 


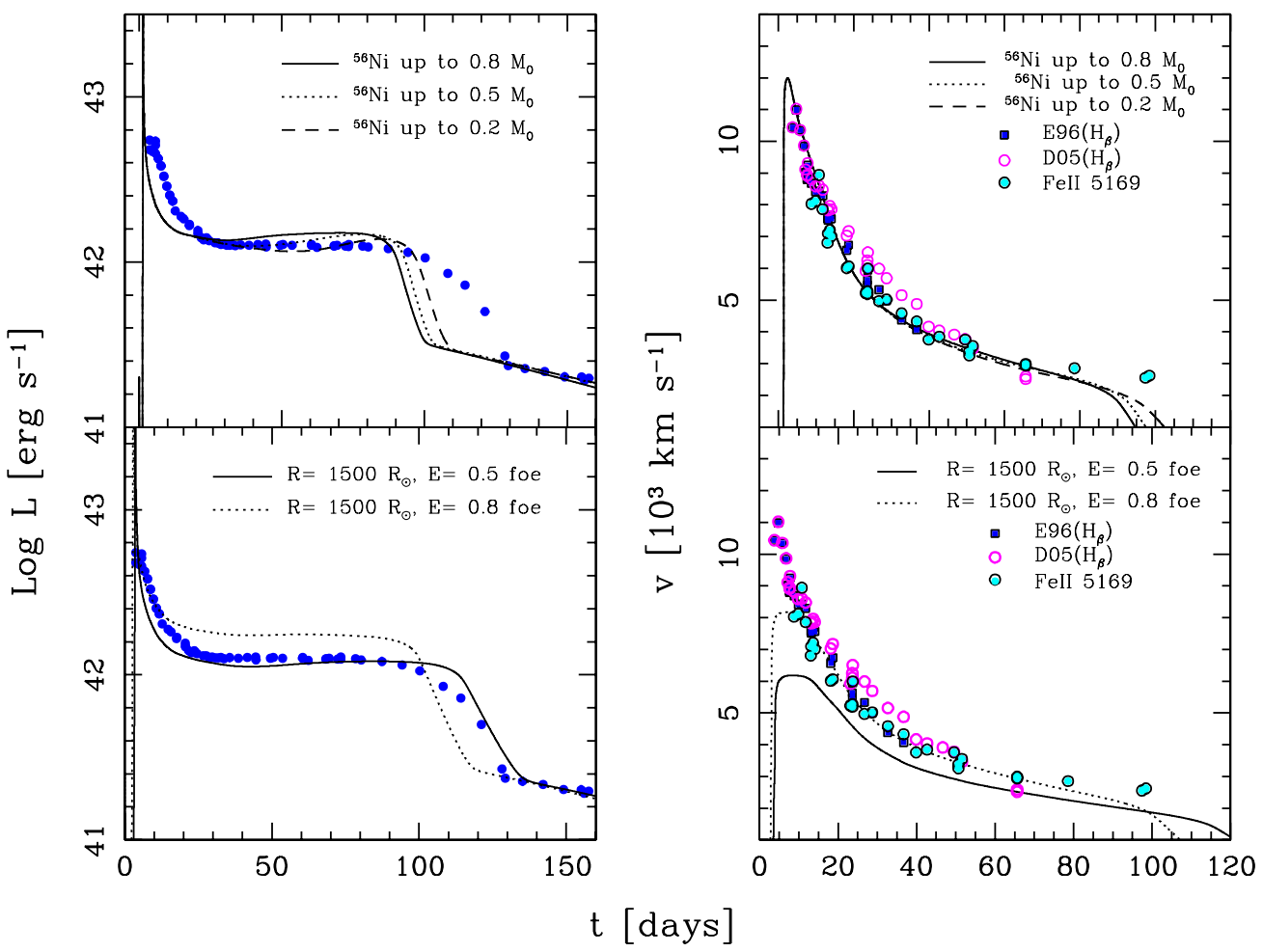

Figure 15. Comparison between models and observations of SN 1999em for a low value of the pre-SNe mass of $M=12 M_{\odot}$ and the same value of ${ }^{56} \mathrm{Ni}$ mass than for the reference model M19R8E1.25NI56. Left panels: bolometric LCs. Right panels: photospheric velocity evolution. Upper panels: models with different mixing of ${ }^{56} \mathrm{Ni}, E=1$ foe, and $R=800 R_{\odot}$. Note that the degree of ${ }^{56} \mathrm{Ni}$ is indicated in each figure as a fraction of the initial mass of the model. Lower panels: models with an initial radius of $R=1500 R_{\odot}$ and two different values for the explosion energy as indicated (corresponding to models m12r15e $08 \mathrm{ni} 56$ and $\mathrm{m} 12 \mathrm{r} 15 \mathrm{e} 05 \mathrm{ni} 56$; see Table 3). Model m12r15e05ni56 reproduces very well the bolometric LC of SN 1999em, but fails to reproduce the photospheric velocity evolution.

(A color version of this figure is available in the online journal.)

${ }^{56} \mathrm{Ni}$ mixing. Table 3 gives a summary of the parameters used. We remark that in all of these models, we have assumed the same value for the ${ }^{56} \mathrm{Ni}$ mass as that of model M19R8E1.25NI56, i.e., $0.056 M_{\odot}$, because this value is required to fit the tail of the LC. Also note that the degree of ${ }^{56} \mathrm{Ni}$ mixing is taken as a fraction of the initial mass of the object in order to compare the different degrees of mixing in a consistent way when models with different initial mass are used. For example, a model with a mixing of ${ }^{56} \mathrm{Ni}$ up to $0.8 M_{0}$ has an equivalent degree of ${ }^{56} \mathrm{Ni}$ mixing as that of model M19R8E1.25NI56.

For the case of $12 M_{\odot}$, we have calculated nine models. We first analyze the effect on the results of the variation of one parameter while keeping the other parameters fixed. In the upper panel of Figure 14, we show the effect on the LC and $v_{\text {ph }}$ of the variation of $E$ for a model with $R=800 R_{\odot}$ and ${ }^{56} \mathrm{Ni}$ mixing up to $0.8 M_{0}$. In the bottom panels of Figure 14, we show the effect of varying $R$, for a model with $E=1$ foe and for the same ${ }^{56} \mathrm{Ni}$ mixing. The sensitivity of the LC and $v_{\text {ph }}$ on the extent of ${ }^{56} \mathrm{Ni}$ mixing is shown in the upper panel of Figure 15 for a model with $E=1$ foe and $R=800 R_{\odot}$.

An examination of these figures yields the following conclusions: (1) higher injected energy produces higher luminosity and a shorter plateau, (2) larger initial radius produces higher luminosity and a longer plateau, (3) more extended ${ }^{56} \mathrm{Ni}$ mixing produces higher luminosity and a shorter plateau, (4) ${ }^{56} \mathrm{Ni}$ mixing and initial radius have a small effect on the $v_{\mathrm{ph}}$ evolution as compared with that of the explosion energy, and (5) as expected, there is no effect of any of these parameters on the tail luminosity. Note that the sensitivity of the LC on $E, R$, and ${ }^{56} \mathrm{Ni}$ mixing is in qualitative concordance with analytic studies by Arnett (1980), Popov (1993), and previous numerical studies by Litvinova \& Nadezhin $(1983,1985)$ and more recently by Young (2004) and Utrobin (2007).

Although we have considered several values of $E, R$, and ${ }^{56} \mathrm{Ni}$ mixing, neither of the models with $M=12 M_{\odot}$ gives a good representation of the observations of SN 1999em. Note that we have also calculated two other models, M12R15E05NI56 and M12R15E08NI56, adopting even higher values of the initial radius. These models are shown in the bottom panel of Figure 15. Despite the good representation of the LC provided by model M12R15E05NI56, it fails to reproduce the observed photospheric velocities. We conclude that it is not possible to reproduce the observations of SN 1999em using models with a pre-SN mass of $M=12 M_{\odot}$. Clearly, masses lower than this value would also fail to match the observations.

For the case of $14 M_{\odot}$, we have calculated 10 models for different combinations of $R, E$, and ${ }^{56} \mathrm{Ni}$ mixing. Specifically, we used three different values of the initial radius: $R=800$, 1000, and $1200 R_{\odot}$, four values of explosion energy: $E=1.1$, 1.0, 0.9, and 0.8 foe, and three different degrees of ${ }^{56} \mathrm{Ni}$ mixing: $0.2,0.5$, and $0.8 M_{0}$ (see Table 3). Figures 16 and 17 show the results of these models. At first sight, we find that these models agree better with the observations than the $12 M_{\odot}$ ones. Therefore, the case of $14 M_{\odot}$ deserves a more detailed analysis. As described below, we chose the grid of parameters based on the known effects of each physical parameter on the LCs and velocities, and trying to reach the best possible agreement with the observations. As noted in Section 3.1, in order to assess the validity of models we will focus on how well they reproduce the observed plateau luminosity and length, and the 


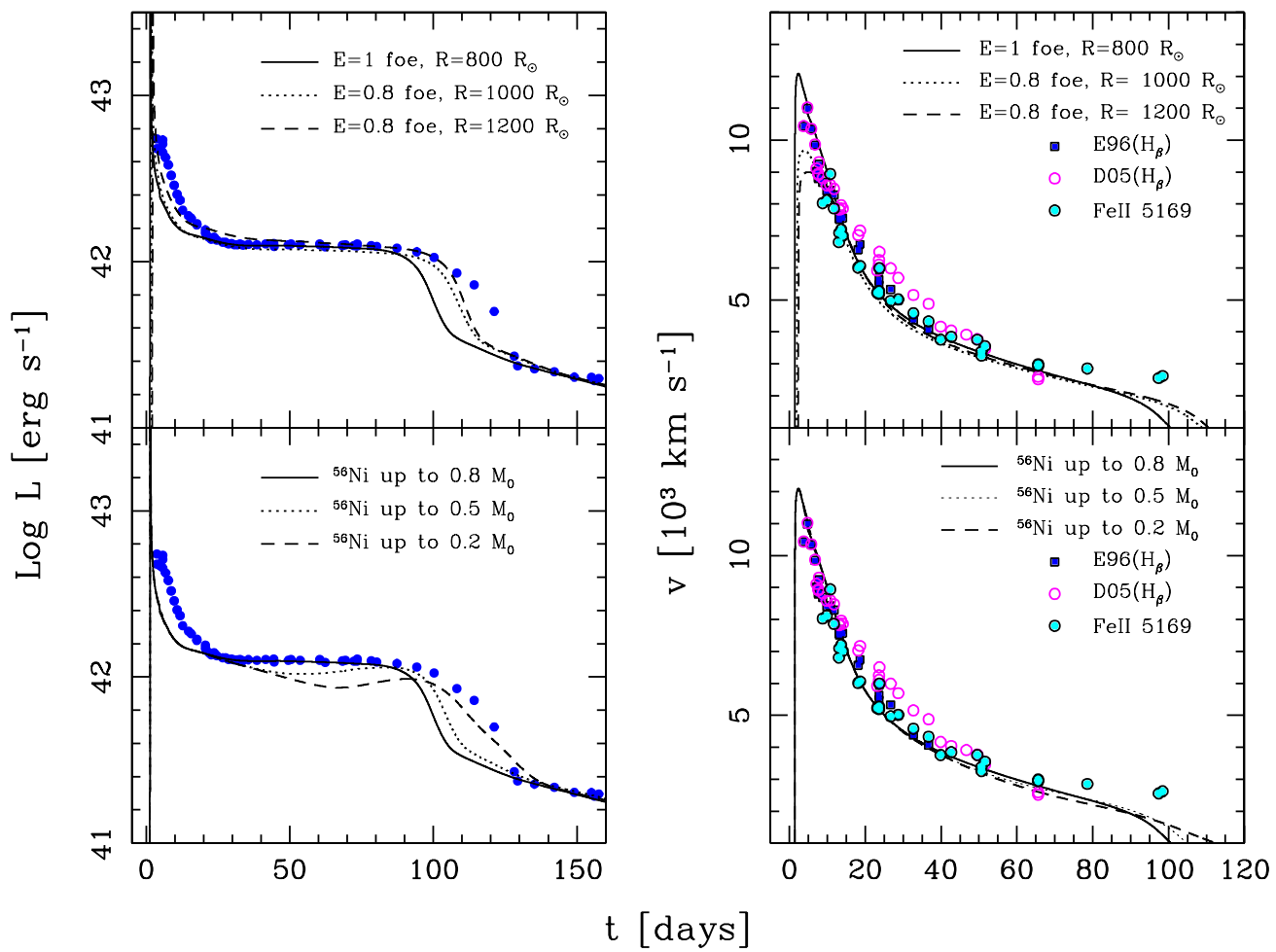

Figure 16. Comparison between models (lines) and observations (points) of SN 1999em for a low value of the pre-SN mass of $M=14 M_{\odot}$ and the same mass of ${ }^{56} \mathrm{Ni}$ as for the reference model M19R8E1.25NI56. Left panels: bolometric LCs. Right panels: photospheric velocity evolution. Upper panels: models M14R8E1Ni56, M14R10E08Ni56, and M14R12E08Ni56 (see Table 3). Lower panels: models with different mixing of ${ }^{56} \mathrm{Ni}, E=1$ foe, and $R=800 R_{\odot}$. Note that the degree of ${ }^{56} \mathrm{Ni}$ is indicated in each panel as a fraction of the initial mass of the model.

(A color version of this figure is available in the online journal.)
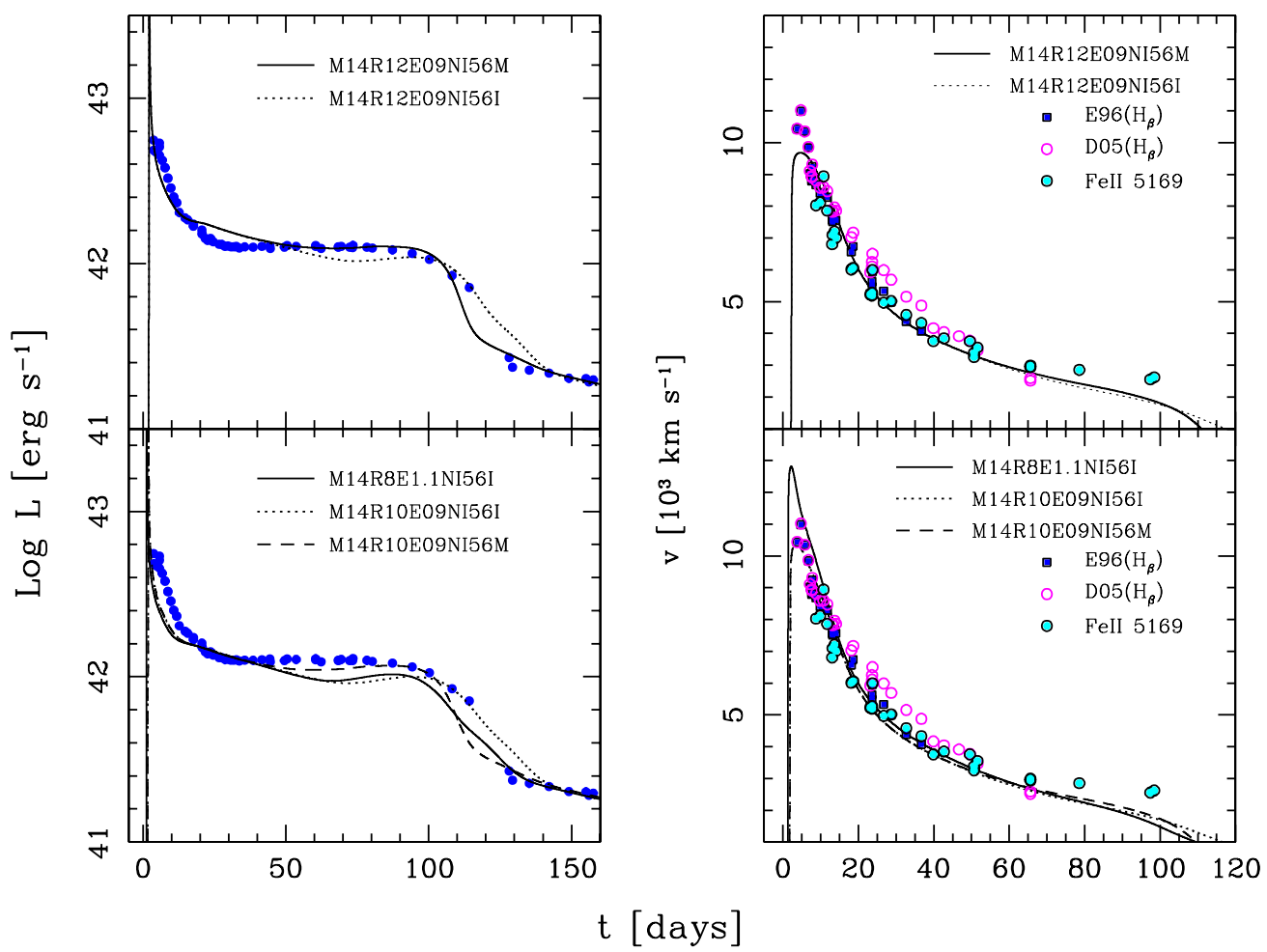

Figure 17. Comparison between models (lines) and observations (points) of SN 1999em for a low value of the pre-SN mass of $M=14 M_{\odot}$ and the same mass of ${ }^{56} \mathrm{Ni}$ as for the reference model M19R8E1.25NI56. Left panels: bolometric LCs. Right panels: photospheric velocity evolution. Upper panels: models M14R8E1.1NI56I, M14R10Е09NI56I, and M14R10Е09NI56M. Lower panels: models M14R12E09NI56M and M14R12E09NI56I (see Table 3).

(A color version of this figure is available in the online journal.) 
early photospheric velocities, but we will disregard differences in the early LC before the plateau phase where the uncertainties in both models and data are the largest.

Our reference model is shown with a solid line in the upper panels of Figure 16 and has the following parameters: $E=1$ foe, $R=800 R_{\odot}$, and $0.8 M_{0}$ of ${ }^{56} \mathrm{Ni}$ mixing. We note that this reference model has the same initial radius and ${ }^{56} \mathrm{Ni}$ mixing as model M19R8E1.25NI56 for $19 M_{\odot}$. The energy has been reduced in order to compensate the effect on the luminosity of using a lower mass. As shown in Figure 16, the reference model produces the correct plateau luminosity and photospheric velocities although the plateau duration is too short as compared with the data. With the aim of remedying this situation while keeping the mass fixed we invoked lower energies and larger radii (dashed and dotted lines). While this served to improve the issue of the plateau length, the comparison with photospheric velocities at early times became poorer, as expected, due to the lower energies. We therefore consider these models to be unlikely. In a further attempt to find a good $14 M_{\odot}$ model, we decided to vary the mixing of ${ }^{56} \mathrm{Ni}$ while keeping the other parameters as in the reference model. The results are shown in the lower panels of Figure 16. We now note that while a reduction of the ${ }^{56} \mathrm{Ni}$ mixing serves to increase the length of the plateau, the shape of the LC and the plateau luminosity drift away from the observations. Thus, we can also discard these models.

Based on the above observations we tested other parameter combinations, as shown in Figure 17. In the upper panels, we show the tests of models with slightly smaller energies and larger radii as compared with the reference model, and two different degrees of ${ }^{56} \mathrm{Ni}$ mixing. While the match of the LC for these models is satisfactory, the problem with the early-time velocities reappears, so we discard these models. In the lower panels of Figure 17, we show further combinations of parameters. Here, we obtain an improvement in the LC while not compromising the agreement in the velocities. Among these models the one called M14R10E09NI56M provides the best match to the LC and velocity data. We therefore conclude that the $14 M_{\odot}$ scenario for SN 1999em cannot be ruled out, although the comparison with the data is not as good as that of the $19 M_{\odot}$ model (see Figures 5 and 6).

\section{CONCLUSIONS}

We have developed a one-dimensional, flux-limited Lagrangian hydrodynamical code useful for modeling bolometric LCs and the photospheric velocity evolution of SNe II-P. The performance of the code was examined by its application to one of the best observed SNe II-P, SN 1999em, obtaining a very good agreement with the observations with the following physical parameters $E=1.25$ foe, $M=19 M_{\odot}, R=800 R_{\odot}$, and $M_{\mathrm{Ni}}=0.056 M_{\odot}$. In our analysis, we found that an extended mixing of ${ }^{56} \mathrm{Ni}$ is needed in order to reproduce a plateau as flat as that shown by the observations of SN 1999em. We note that the plateau phase, at least in the case of this SN, is powered by the energy deposited by the SW and released by the recombination process, plus some extra energy deposited by the radioactive material.

Our model for SN 1999em required extensive mixing of ${ }^{56} \mathrm{Ni}$ into the envelope. This has also been strongly suggested by the modeling of the observations of SN 1987A (see Shigeyama et al. 1988; Woosley et al. 1988; Arnett 1988; Blinnikov et al. 2000, among others). In addition, Baklanov et al. (2005) have also invoked extensive ${ }^{56} \mathrm{Ni}$ mixing to model SN 1999em. Young (2004) explored the effect on the LCs of SNe II of the variation of several parameters and found, in concordance with our study, that between 50 and 120 days, the LC is powered by the energy deposited by the SW in combination with energy deposited by gamma rays when an extended mixing of ${ }^{56} \mathrm{Ni}$ is used.

We compare our results for SN 1999em with two hydrodynamical studies of this object previously given by BBP05 and U07. Although there are differences in the physical parameters used in each work, we believe that the results are remarkably consistent considering the differences in distance, explosion time, mass cut, the observational data employed, and the very different prescriptions for the radiative transfer. Note that the physical parameters used in our calculations are intermediate between those estimated by BBP05 and U07. Although it is satisfactory that our code provides good fits to the observations with physical parameters similar to those obtained from more sophisticated calculations, we note that given the differences among the three approaches, the comparison does not help to assess the validity of our assumptions nor the predictive power of the methods.

We have also explored the feasibility of fitting the observations of SN 1999em with low-mass models, consistent with the upper limits obtained through pre-SN imaging in connection with stellar evolution models. We were not able to find a set of physical parameters that reproduce well the observations when assuming pre-SN masses of $\leqslant 12 M_{\odot}$. On the other hand, models with $14 M_{\odot}$ cannot be fully discarded. Specifically, we found one model with the following parameters: $E=0.9$ foe, $R=1000 R_{\odot}$, and $0.8 M_{0}$ of ${ }^{56} \mathrm{Ni}$ mixing which provides a reasonable fit to the observations, although not as good as our favorite model of $19 M_{\odot}$. We remark that even if the exact values of the progenitor mass may be affected by the choice of parametric initial models, this comparative analysis favors models with total masses larger than $14 M_{\odot}$.

The fact that models with different masses agree reasonably well with the observations tells us that there is a degree of degeneracy among the physical parameters which must be quantified if one wants to assess the precision to which such parameters can be determined using hydrodynamical calculations. We plan to continue investigating this issue with our code.

Additional information such as modeling of spectra can help in discriminating among possible scenarios. On the other hand, one way to reduce the number of free parameters would be to use initial models from stellar evolution calculations. However, the use of evolutionary initial models introduces unobserved features in the resulting LCs (see Section 2.4 and also Utrobin \& Chugai 2008) probably caused by sharp boundaries between layers with different chemical compositions and/or the steep density jump between the helium core and the hydrogen envelope. With our parametric density and chemical profiles we were able to smooth the transitions and thereby obtain smooth LC. The disadvantage of this approach is that parametric initial models are less directly connected with actual properties of the progenitor star.

As has been previously proposed, we suggest that a possible reason for the need of invoking ad hoc initial models is the fact that the calculations of the shock propagation are done in one dimension. Therefore, known effects that cause mixing, such as $\mathrm{R}-\mathrm{T}$ instabilities, cannot be taken into account. Note that even if $\mathrm{R}-\mathrm{T}$ instabilities produce mixing behind the shock, the effect that is observed in our modeled LCs is related to the structure 
that is left behind the passage of the shock once it has reached the surface. Such structure is revealed as the recombination front recedes into the ejecta. Therefore, we think that it is important to attempt to quantify the effect of the R-T instability on the mixing and density structure in one dimension in order to check if this effect can explain the type of initial distributions and mixing assumed in our calculations.

The remarkably good agreement with the observation of SN 1999em and the consistency found with previous hydrodynamical studies of this SN using more sophisticated codes give us confidence in our attempt to model SNe II-P, at least during the plateau phase and early evolution of the radioactive tail phase, despite the simplifications assumed in our calculation. This work is the starting point for the analysis of the physical parameters of our sample of 33 SNe II-P that will be done in a forthcoming paper with the aim of improving our understanding of these objects.

The authors gratefully acknowledge the helpful conversations with Sergei Blinnikov, Ken'ichi Nomoto, Nozomu Tominaga, and Alejandro Clocchiatti. A special thanks to Gastón Folatelli for his support and generous revision of the text. M.C.B. acknowledges support from MECESUP UCH0118 program and the World Premier International Research Center Initiative, MEXT, Japan. O.B. is a member of the Carrera del Investigador Científico, Comisión de Investigaciones Científicas de la Provincia de Buenos Aires, Argentina. M.H. obtained support from proyecto FONDECYT (grant 1060808), Millennium Center for Supernova Science through grant P06-045-F, Centro de Astrofísica FONDAP 15010003, and Center of Excellence in Astrophysics and Associated Technologies (PFB 06).

\section{REFERENCES}

Alexander, D. R., \& Ferguson, J. W. 1994, ApJ, 437, 879

Arnett, D. (ed.) 1996, Supernovae and Nucleosynthesis: An Investigation of the History of Matter, from the Big Bang to the Present (Princeton, NJ: Princeton Univ. Press)

Arnett, W. D. 1980, ApJ, 237, 541

Arnett, W. D. 1988, ApJ, 331, 377

Baklanov, P. V., Blinnikov, S. I., \& Pavlyuk, N. N. 2005, Astron. Lett., 31, 429

Barbon, R., Ciatti, F., \& Rosino, L. 1979, A\&A, 72, 287

Baron, E., Hauschildt, P. H., Nugent, P., \& Branch, D. 1996, MNRAS, 283, 297

Baron, E., et al. 2000, ApJ, 545, 444

Bersten, M. C., \& Hamuy, M. 2009, ApJ, 701, 200

Blinnikov, S. I., \& Bartunov, O. S. 1993, A\&A, 273, 106

Blinnikov, S. I., Eastman, R., Bartunov, O. S., Popolitov, V. A., \& Woosley, S. E. 1998, ApJ, 496, 454

Blinnikov, S., Lundqvist, P., Bartunov, O., Nomoto, K., \& Iwamoto, K. 2000, ApJ, 532, 1132

Burrows, A. 1991, in The 10th Santa Cruz Workshop in Astronomy and Astrophysics, Supernovae, ed. S. E. Woosley (New York: Springer), 393

Burrows, A., Livne, E., Dessart, L., Ott, C. D., \& Murphy, J. 2006, New Astron. Rev., 50, 487

Chevalier, R. A. 1976, ApJ, 207, 872

Chevalier, R. A., Fransson, C., \& Nymark, T. K. 2006, ApJ, 641, 1029

Chevalier, R. A., \& Soker, N. 1989, ApJ, 341, 867

Chieffi, A., Domínguez, I., Höflich, P., Limongi, M., \& Straniero, O. 2003, MNRAS, 345, 111

Christy, R. F. 1967, in Methods in Computational Physics, Vol. 7, Astrophysics, ed. B. Alder, S. Fernbach, \& M. Rotenberg (New York: Academic), 191

Deputovich, A. Y., \& Nadyozhin, D. K. 1999, Astron. Lett., 25, 649

Dessart, L., \& Hillier, D. J. 2005, A\&A, 437, 667

Dessart, L., \& Hillier, D. J. 2008, MNRAS, 383, 57

Dessart, L., Livne, E., \& Waldman, R. 2010, MNRAS, 408, 827

Dotani, T., Hayashida, K., Inoue, H., Itoh, M., \& Koyama, K. 1987, Nature, 330,230
Eastman, R. G., Schmidt, B. P., \& Kirshner, R. 1996, ApJ, 466, 911

Elmhamdi, A., Chugai, N. N., \& Danziger, I. J. 2003, A\&A, 404, 1077

Ensman, L., \& Burrows, A. 1992, ApJ, 393, 742

Falk, S. W., \& Arnett, W. D. 1977, ApJS, 33, 515

Grassberg, E. K., Imshennik, V. S., \& Nadyozhin, D. K. 1971, Ap\&SS, 10, 28

Haas, M. R., Erickson, E. F., Lord, S. D., Hollenbach, D. J., Colgan, S. W. J., \& Burton, M. G. 1990, ApJ, 360, 257

Hamuy, M. 2001, PhD thesis, Univ. Arizona

Hamuy, M. 2003, ApJ, 582, 905

Hamuy, M., \& Pinto, P. A. 2002, ApJ, 566, L63

Heger, A., Fryer, C. L., Woosley, S. E., Langer, N., \& Hartmann, D. H. 2003, ApJ, 591,288

Herzig, K., El Eid, M. F., Fricke, K. J., \& Langer, N. 1990, A\&A, 233, 462

Hillebrandt, W. 1994, in Proc. 54th Éoledété de Physique Théorique, Supernovae, ed. S. A. Bludman, R. Mochkovitch, \& J. Zinn-Justin (NATO ASI Ser. C; Amsterdam: Elsevier), 251

Hoflich, P. 1991, in The 10th Santa Cruz Workshop in Astronomy and Astrophysics, Supernovae, ed. S. E. Woosley (New York: Springer), 415

Iglesias, C. A., \& Rogers, F. J. 1996, ApJ, 464, 943

Imshenik, V. S., \& Nadezhin, D. K. 1965, SvA, 8, 664

Janka, H.-T., Langanke, K., Marek, A., Martínez-Pinedo, G., \& Müller, B. 2007, Phys. Rep., 442, 38

Jones, M. I., et al. 2009, ApJ, 696, 1176

Kane, J., Arnett, D., Remington, B. A., Glendinning, S. G., Bazán, G., Müller, E., Fryxell, B. A., \& Teyssier, R. 2000, ApJ, 528, 989

Karp, A. H., Lasher, G., Chan, K. L., \& Salpeter, E. E. 1977, ApJ, 214, 161

Kasen, D., \& Woosley, S. E. 2009, ApJ, 703, 2205

Leonard, D. C., \& Filippenko, A. V. 2001, PASP, 113, 920

Leonard, D. C., \& Filippenko, A. V. 2005, in ASP Conf. Ser. 342, 1604-2004: Supernovae as Cosmological Lighthouses, ed. M. Turatto et al. (San Francisco, CA: ASP), 330

Leonard, D. C., Kanbur, S. M., Ngeow, C. C., \& Tanvir, N. R. 2003, ApJ, 594, 247

Levermore, C. D., \& Pomraning, G. C. 1981, ApJ, 248, 321

Li, W. D. 1999, IAU Circ., 7294, 1

Litvinova, I. I., \& Nadezhin, D. K. 1983, Ap\&SS, 89, 89

Litvinova, I. Y., \& Nadezhin, D. K. 1985, Sov. Astron. Lett., 11, 145

Montes, M. J., \& Wagoner, R. V. 1995, ApJ, 445, 828

Mueller, E., Fryxell, B., \& Arnett, D. 1991, A\&A, 251, 505

Nadyozhin, D. K. 2003, MNRAS, 346, 97

Popov, D. V. 1993, ApJ, 414, 712

Rogers, F. J., Swenson, F. J., \& Iglesias, C. A. 1996, ApJ, 456, 902

Shigeyama, T., \& Nomoto, K. 1990, ApJ, 360, 242

Shigeyama, T., Nomoto, K., \& Hashimoto, M. 1988, A\&A, 196, 141

Shigeyama, T., Nomoto, K., Hashimoto, M., \& Sugimoto, D. 1987, Nature, 328, 320

Smartt, S. J. 2009, ARA\&A, 47, 63

Smartt, S. J., Eldridge, J. J., Crockett, R. M., \& Maund, J. R. 2009, MNRAS, 395, 1409

Smartt, S. J., Maund, J. R., Hendry, M. A., Tout, C. A., Gilmore, G. F., Mattila, S., \& Benn, C. R. 2004, Science, 303, 499

Sobolev, V. V. 1980, Astrofizika, 16, 695

Sorokina, E. I., \& Blinnikov, S. I. 2002, in Proc. 11th Workshop on Nuclear Astrophysics, ed. W. Hillebrandt \& E. Müller (Garching b. München: MaxPlanck-Institut für Astrophysik), 57

Sutherland, P. G., \& Wheeler, J. C. 1984, ApJ, 280, 282

Swartz, D. A., Sutherland, P. G., \& Harkness, R. P. 1995, ApJ, 446, 766

Swartz, D. A., Wheeler, J. C., \& Harkness, R. P. 1991, ApJ, 374, 266

Umeda, H., \& Nomoto, K. 2005, ApJ, 619, 427

Utrobin, V. 1993, A\&A, 270, 249

Utrobin, V. P. 2004, Astron. Lett., 30, 293

Utrobin, V. P. 2007, A\&A, 461, 233

Utrobin, V. P., \& Chugai, N. N. 2008, A\&A, 491, 507

Utrobin, V. P., \& Chugai, N. N. 2009, A\&A, 506, 829

Van Dyk, S. D., Li, W., \& Filippenko, A. V. 2003, PASP, 115, 1289

Von Neumann, J., \& Richtmyer, R. D. 1950, Appl. Phys., 21, 232

Woosley, S. E. 1988, ApJ, 330, 218

Woosley, S. E., Pinto, P. A., \& Ensman, L. 1988, ApJ, 324, 466

Woosley, S. E., \& Weaver, T. A. 1994, in Proc. 54th Éoledété de Physique Théorique, Supernovae, ed. S. A. Bludman, R. Mochkovitch, \& J. ZinnJustin (NATO ASI Ser. C; Amsterdam: Elsevier), 63

Young, T. R. 2004, ApJ, 617, 1233

Young, T. R., \& Branch, D. 1989, ApJ, 342, L79 